\title{
Italian consensus on diagnosis and treatment of differentiated thyroid cancer: joint statements of six Italian societies
}

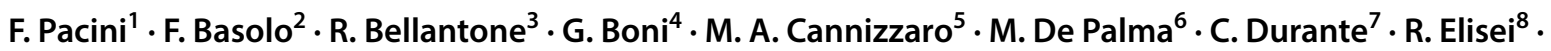 \\ G. Fadda ${ }^{9} \cdot$ A. Frasoldati ${ }^{10} \cdot$ L. Fugazzola ${ }^{11,12} \cdot$ R. Guglielmi $i^{13}$. C. P. Lombardi ${ }^{3} \cdot$ P. Miccoli ${ }^{2} \cdot$ E. Papini $^{13} \cdot$ G. Pellegriti ${ }^{14}$. \\ L. Pezzullo ${ }^{15} \cdot$ A. Pontecorvi $^{16} \cdot$ M. Salvatori $^{17} \cdot$ E. Seregni $^{18} \cdot$ P. Vitti $^{8}$
}

Received: 27 January 2018 / Accepted: 31 March 2018 / Published online: 4 May 2018

(c) Italian Society of Endocrinology (SIE) 2018

\begin{abstract}
Background Thyroid nodules are a common clinical problem, and differentiated thyroid cancer is becoming increasingly prevalent.

Methods Six scientific Italian societies entitled to cure thyroid cancer patients (the Italian Thyroid Association, the Medical Endocrinology Association, the Italian Society of Endocrinology, the Italian Association of Nuclear Medicine and Molecular Imaging, the Italian Society of Unified Endocrine Surgery and the Italian Society of Anatomic Pathology and Diagnostic Cytology) felt the need to develop a consensus report based on significant scientific advances occurred in the field.

Objective The document includes recommendations regarding initial evaluation of thyroid nodules, clinical and ultrasound criteria for fine-needle aspiration biopsy, initial management of thyroid cancer including staging and risk assessment, surgical management, radioiodine remnant ablation, and levothyroxine therapy, short-term and long-term follow-up strategies, and management of recurrent and metastatic disease. The objective of this consensus is to inform clinicians, patients, researchers, and health policy makers about the best strategies (and their limitations) relating to the diagnosis and treatment of differentiated thyroid cancer.
\end{abstract}

Keywords Thyroid nodules · Thyroid cancer · Thyroid surgery $\cdot$ Radioiodine

$\begin{array}{ll}\text { Abbreviations } \\ \text { PTMC } & \text { Papillary thyroid microcarcinoma } \\ \text { DTC } & \text { Differentiated thyroid cancer } \\ \text { Tg } & \text { Thyroglobulin } \\ \text { TgAb } & \text { Anti-thyroglobulin antibodies } \\ \text { rhTSH } & \text { Recombinant human TSH } \\ \text { WBS } & \text { Whole-body scan } \\ \text { RAI } & \text { Radioactive iodine } \\ \text { US } & \text { Ultrasound } \\ \text { FNA(C) } & \text { Fine-needle aspiration (cytology) }\end{array}$

Joint statements of six Italian societies: The Italian Thyroid Association (AIT), the Medical Endocrinology Association (AME), the Italian Society of Endocrinology (SIE), the Italian Association of Nuclear Medicine and Molecular Imaging (AIMN), the Italian Society of Unified Endocrine Surgery (SIUEC) and the Italian Society of Anatomic Pathology and Diagnostic Cytology (SIAPEC).

F. Pacini

pacini8@unisi.it

Extended author information available on the last page of the article
CCND Central compartment neck dissection

LID Low-iodine diet

MTA Maximum tolerated activity (of radioiodine)

CNO No clinical evidence of lymph nodes

\section{Introduction}

Although rare among human malignancies $(<1 \%)$, thyroid cancer of the follicular epithelium is the most frequent endocrine cancer, accounting for about $5 \%$ of thyroid nodules. The latter are very frequent in the general population, and according to the method of detection and the subject's 
age, their prevalence may approach $20-50 \%$ of the general population, thus representing a daily issue in endocrine clinics. In recent decades, differentiated thyroid cancer (DTC), mainly papillary, has emerged as the most rapidly increasing human cancer worldwide. It is estimated that by the year 2020 , thyroid cancer will be the second most frequent cancer in women. The increase is believed to be mainly due to a screening effect after the introduction of neck ultrasonography in daily practice performed for thyroid diseases but also for other unrelated conditions. Indeed, the large majority of thyroid cancers detected nowadays is constituted by the discovery of non-palpable small tumors escaping recognition in the previous years. On the other hand, the rate of cancerrelated mortality does not seem to be at increase, although this aspect is still controversial. Based on this consideration, it is time to tailor diagnostic and therapeutic strategies for thyroid nodules and cancer according to the individual risk of recurrence or death. Management of the disease requires a multidisciplinary approach, including endocrinology, nuclear medicine, oncology, endocrine surgery, pathology, and even general practice operating in different settings not always equipped with the appropriate services (such as specialized centers, general hospitals, and peripheral centers). Diagnostic and treatment tools have significantly improved in recent years, thus allowing for less invasive and more comfortable procedures for the patients. Altogether, these considerations dictate the need for applying the more effective, less invasive, and less expensive procedures able to guarantee the best management and the best quality of life for a disease that albeit having an intrinsic low mortality requires life-long follow-up.

Several countries have developed their own guidelines or consensus reports, based on consolidated experience and cultural attitude of the country. Nevertheless, they differ in several, sometime important aspects. Following the spirit of a concrete cultural and scientific integration among different actors, the present document has been implemented and endorsed by six scientific societies entitled to cure thyroid cancer patients: the Italian Thyroid Association (AIT), the Medical Endocrinology Association (AME), the Italian Society of Endocrinology (SIE), the Italian Association of Nuclear Medicine and Molecular Imaging (AIMN), the Italian Society of Unified Endocrine Surgery (SIUEC) and the Italian Society of Anatomic Pathology and Diagnostic Cytology (SIAPEC).

The text is not intended to represent classical guidelines, but rather a collection of practical statements on selected relevant issues in the management of thyroid nodules and cancer, based on an adaptation of current guidelines (mainly the American Thyroid Association Guidelines) [1] according to the Italian situation and the expert opinion of the multidisciplinary panel.

\section{Actions}

For the implementation of the consensus, each of the six Societies selected its own experts for a total of 21 experts, including one coordinator. They met in ad hoc meeting that took place in Pisa on May 2016, where the relevant diagnostic and therapeutic issues to be covered were identified. Each item was attributed to two members of the panel, who wrote the first draft of the statement, and then discussed in periodical plenary teleconferences until an agreement was reached. At this point, the panel met again in a meeting for final consensus which took place in the Pontificia Accademia delle Scienze of the Vatican state, 15-16 September 2017. Experts were advised to base their statements on clinical and scientific evidence whenever available in the current literature and on their own personal experience.

\section{List of items}

Item 1 Selection of thyroid nodules to be submitted to cytological evaluation.

Item 2 Based on cytology result, when thyroid surgery is indicated?

Item 3 What is the role of pre-operative staging with diagnostic imaging and laboratory tests?

Item 4 When surgery is indicated which strategy should be performed: total thyroidectomy or lobo-isthmectomy?

Item 5 No intervention for papillary thyroid microcarcinoma.

Item 6 Indication for completion thyroidectomy after lobectomy.

Item 7 The role of central and lateral compartment neck dissection in the management of differentiated thyroid carcinoma (DTC).

Item 8 The need for a complete histological and cytological report.

Item 9 Post-surgical thyroid ablation with radioiodine: routine or selective indication.

Item 10 Preparation for thyroid ablation and selection of the most appropriate activity.

Item 11 Informing the patient treated with radioactive iodine on safety measures.

Item 12 Follow-up after initial treatment (surgery and radioiodine): when and how.

Item 13 Risk stratification incorporating the response to initial treatment.

Item 14 Follow-up of patients with excellent response to initial therapy.

Item 15 Follow-up of patients with persistent structural disease or biochemical disease.

Item 16 Follow-up of patients treated with lobo-isthmectomy. 
Item 17 Is there an indication for rhTSH in the preparation of patients undergoing radioiodine therapy for metastatic disease?

Item 18 Radioiodine treatment of metastatic disease: standard activities vs. dosimetry, maximal cumulative activity, and frequency of treatment courses.

Item 19 "Empiric" treatment with radioiodine in Tg positive/WBS negative patients: still an indication?

Item 20 Adverse events of radioiodine therapy.

Item 21 Definition of RAI resistant disease.

Item 22 What is the role of systemic therapy (kinase inhibitors and conventional chemotherapy) in treating metastatic DTC?

Item 23 L-Thyroxine therapy after total thyroidectomy or lobectomy.

Item 24 Legal issue in the management of thyroid cancer.

\section{Item 1: Selection of thyroid nodules to be submitted to cytological evaluation}

\section{Recommendation statement}

In thyroid US reports, a conclusive score that stratifies thyroid nodules on the basis of risk of malignancy should be provided according to the following Ultrasound (US) Rating of the Risk of Malignancy:

Class 1. Low-risk thyroid lesion:

Purely cystic nodules have virtually no risk. Mostly cystic $(>80 \%)$ nodules with reverberating artifacts have very low risk $(<1 \%)$.

Spongiform, isoechoic or hyperechoic nodules not associated with suspicious US findings (expected risk of malignancy, about $1 \%$ ).

Class 2. Intermediate-risk thyroid lesion:

Slightly hypo- or isoechoic nodules with ovoid-to-round shape and smooth or ill-defined margins. May be present: intranodular vascularization, macro- or continuous rim calcifications, increased stiffness at elastography or hyperechoic spots of uncertain significance (expected risk of malignancy, 5-15\%).

Class 3. High-risk thyroid lesion:

Nodules with at least one of the suspicious findings: marked hypoechogenicity, spiculated or microlobulated margins, micro-calcifications, taller-than-wide shape, extrathyroidal growth or lymphadenopathy (expected risk of malignancy, $50-90 \%$, according to the presence of one or more suspicious findings).

\section{Recommendation for FNA}

\section{Low-US-risk thyroid lesions (Class 1)}

(a) US-guided FNA is recommended for nodules $\geq 20 \mathrm{~mm}$ in diameter only when symptomatic, increasing in size, associated with high-risk factors, or before surgery or local percutaneous therapy.

(b) Hyperfunctioning thyroid nodules: FNA is usually not recommended.

Intermediate-US-risk thyroid lesions (Class 2)

(a) US guided FNA is recommended for nodules $\geq 20 \mathrm{~mm}$. For smaller nodules, clinical monitoring is indicated.

\section{High-US-risk thyroid lesions (Class 3)}

(a) Diameter 5-9 mm: either US-guided FNA sampling or US monitoring on the basis of clinical setting and patient preference. US-guided FNA is recommended for subcapsular, posterior or paratracheal lesions, or in case of suspicious lymph nodes, extrathyroid spread, clinical thyroid cancer risk factors.

(b) Diameter $\geq 10 \mathrm{~mm}$ : US-guided FNA is recommended.

\section{Narrative of the recommendation}

Nowadays, most thyroid nodules are detected incidentally in euthyroid subjects and are not associated with clinical symptoms. Thus, the major task in their management is to exclude the minority of cases (about 5\%) that correspond to a malignant lesion. Thyroid US, clinical features and patient age are the basis for selecting thyroid nodules that deserve US-guided fine-needle aspiration (FNA) cytology [1]. Thyroid scintigraphy is usually not required and should be performed only in those patients with low TSH levels to demonstrate potential areas of functional autonomy, although in sporadic cases, thyroid autonomy may be present even with low-normal TSH values, particularly in multinodular goiters. Currently, US features are more relevant than the size of the nodule to define the risk of cancer and the strength of indication to FNA.

In the selection of nodules for US-guided FNA, however, a correct balance between the risk of a missed diagnosis of low-risk cancer and that of a large-scale use of inappropriate invasive procedures should be considered.

A higher probability of malignancy is associated with the following findings: nodule height greater than width, microcalcifications, irregular margins, and marked hypoechogenicity. Solid nodule structure, intranodular vascular 
signals, and mild hypoechogenicity have a lower predictive value. As in a part of thyroid carcinomas, the US signs predictive of malignancy are lacking, and the absence of clearly suspicious features is not diagnostic for a benign lesion.

\section{Size and number}

Nodule volume is not a predictive factor for malignancy even if the risk of cancer and of a potentially more advanced disease is slightly higher in nodules $>4 \mathrm{~cm}$. Thus, small suspicious nodules $(<10 \mathrm{~mm})$ may be considered for either US-guided FNA or US follow-up, while incidental thyroid lesions with a diameter $\leq 5 \mathrm{~mm}$ should only be followed over time.

Volume increase is not indicative of malignancy. Rapid growth is observed only in the infrequent highly aggressive tumors and is generally associated with suspicious US findings.

Nodule volume assessment by the ellipsoid formula (longitudinal diameter $\times$ transverse diameter $\times$ anteroposterior diameter $\times \pi / 6$ ) should be used for follow-up. A $20 \%$ increase in two diameters or $50 \%$ of the volume should be used as the minimum threshold for significant nodule growth.

\section{Structure and echogenicity}

Most carcinomas are visualized as hypoechoic solid lesions, but about half of benign thyroid nodules show a similar appearance. Therefore, mild hypoechogenicity is a sensitive but poor specific predictor of malignancy. Marked hypoechogenicity is a highly suspicious finding.

Predominantly cystic thyroid nodules are almost never malignant, although occasional cases have been reported.

Iso- or hyperechoic thyroid lesions characterized by the aggregation of multiple microcystic components that comprise more than $50 \%$ of the volume ("spongiform nodules") are nearly always benign.

\section{Margins and shape}

The majority of benign thyroid lesions have a regular round to oval profile. A taller-than-wide shape and the presence of ill-defined margins deserve attention, while irregular, spiculated, or lobulated contours are highly specific signs of malignancy.

A regular hypoechoic halo is a typical finding in benign nodules, while a thick, irregular or incomplete, hypoechoic halo due to inflammatory or necrotic changes may be observed in differentiated thyroid carcinoma.

\section{Calcifications}

Micro-calcifications are revealed as $\leq 1 \mathrm{~mm}$ hyperechoic spots without posterior shadowing (unless in cluster). The specificity for malignancy of true micro-calcifications is elevated, but, unfortunately, their sensitivity is rather low. Intranodular hyperechoic spots that are not due with certainty to micro-calcifications should be reported as "hyperechoic spots of uncertain significance" to prevent an inappropriate upgrading of the risk of PTC.

Macrocalcifications are generally due to regressive changes, but should be considered as a potential risk factor.

Peripheral ("rim") calcifications are sometimes found in nodular goiters. A discontinuity of the egg-shell structure due to extrusive growth of hypoechoic tissue may be predictive of malignancy.

\section{Vascularity}

Color- and power-Doppler evaluation of thyroid nodules provides only complementary data. Malignant lesions (mainly, follicular thyroid carcinoma) may demonstrate a rich intranodular pattern, but this finding may be present in benign nodules as well. A scanty vascularity is observed in most benign nodules, but PTMC may appear as avascular lesions as well.

\section{Elastography}

The stiffness of thyroid nodules during the delivery of an impulse by the US probe has a good sensitivity for thyroid carcinoma with a high negative predictive value. Elastography may be an additional complementary diagnostic tool used in some centers, but current evidence does not allow to recommend its use in routine practice.

\section{Further suspicious US findings}

Growth of thyroid nodules beyond the capsule and infiltration of the trachea or recurrent laryngeal nerves are infrequent but threatening features that warrant cytologic evaluation.

Enlarged lymph nodes with cystic changes, micro-calcifications, and increased echogenicity (thyroid tissue-like appearance) are highly suspicious. Rounded appearance, chaotic hypervascularity, and the absence of hilum are less specific US features of malignancy [2]. 
Item 2: Based on cytology result, when is thyroid surgery indicated?

\section{Recommendation statement}

Non-diagnostic nodules (TIR 1)

(a) In nodules solid at US, repeat FNA is recommended. Clinical and US follow-up may be considered for nondiagnostic cystic or predominantly cystic nodules (TIR 1C) without suspicious clinical or US features.

(b) Consider diagnostic surgery for persistently non-diagnostic solid nodules if suspicious clinical and US features are present and the size is greater than $1 \mathrm{~cm}$.

\section{Benign nodules (TIR 2)}

(a) Most TIR 2 nodules do not require treatment.

(b) Surgery is recommended in the presence of compressive symptoms.

(c) The preferred extent of surgical resection for benign uninodular goiter is lobectomy, while for bilateral multinodular goiter is total thyroidectomy.

\section{Low-risk indeterminate nodules (TIR 3A)}

(a) We recommend repeat FNA and consultation with an experienced cytopathologist.

(b) Consider conservative management in the case of favorable clinical and US criteria, while thyroid lobectomy may be considered in the other cases. Total thyroidectomy may be considered depending on the clinical setting, coexistence of contralateral nodules and patient preference.

\section{High-risk indeterminate lesions (TIR 3B)}

(a) Surgery is recommended in most cases

(b) Thyroid lobo-isthmectomy is recommended. Total thyroidectomy may be performed, depending on the clinical situation, coexistence of other thyroid nodules, and patient preference.

(c) Consider close clinical follow-up in a minority of cases with favorable clinical and US features, after discussion of treatment options with the patient.

(d) For both TIR3A and TIR3B categories, mutational analyses (at least BRAF and RAS genes), if accessible, is of added value for the diagnosis of malignancy, when mutations are detected.
Suspicious nodules (TIR 4)

(a) Surgery is recommended in most cases (see TIR 5). Additional techniques (molecular biology) for a better characterization may be considered in selected cases.

\section{Malignant nodules (TIR 5)}

(a) Surgery is recommended. The extent of surgery is total thyroidectomy in most cases, but lobectomy may be considered for intrathyroidal, uni-or multiple unilateral small nodules.

(b) A close follow-up without surgery may be considered in selected low-risk patients (see "Item 5") with small nodules and in intermediate-risk fragile subjects, after careful discussion with the patient. The rationale for this statement is based on the very favorable outcome even without surgery.

(c) For anaplastic or medullary thyroid carcinoma, metastatic lesions, and primary thyroid lymphoma further multidisciplinary diagnostic work-up is recommended.

\section{Narrative of the recommendation}

The decision for surgery resides primarily on the result of cytology, but also on other considerations such as the size of the nodule, the age of the patient and the presence of comorbidities. In this consensus, the Italian classification for cytology [3] is followed that subdivides TIR 3 in two categories TIR $3 \mathrm{~A}$ and TIR 3B, with different risks of malignancy, similar to the subdivision in AUS/FLUS-FN/SFN of the Bethesda [4] and to the Thy 3 " $a$ " and " $f$ " of British Thyroid Association systems [5]. Yet, at variance with these two reference systems, in the Italian classification, the category TIR 3B includes those cases with "mild/focal nuclear atypia' that have a higher risk of malignancy.

\section{Non-diagnostic nodules (TIR 1)}

Non-diagnostic FNA specimens often result from cystic (TIR1C) or mixed nodules that are mostly benign. However, solid nodules sometimes yield repeatedly non-diagnostic results by FNA. These nodules should be considered for surgery in the presence of clinical or US suspicious findings or in the case of growth ( $>20 \%$ in 2 dimensions). Consider core needle biopsy in experienced centers.

\section{Benign nodules (TIR 2)}

Most nodules with benign cytology do not require treatment. The expected risk of malignancy is below 3\%. Surgery is 
indicated in nodules of large size $(>4 \mathrm{~cm})$ in young patients and in the presence of pressure symptoms. Surgery should also be considered in nodules that develop suspicious US changes or increase in volume and become symptomatic. The preferred extent of resection is lobectomy for benign uninodular goiter and total thyroidectomy for bilateral multinodular goiter. Surgery may be considered for benign hyperfuctioning thyroid nodules $>3 \mathrm{~cm}$, especially in young patients. Local treatment techniques (laser or radiofrequency ablation) may be considered for selected cystic lesions or symptomatic solid nodules without suspicious clinical and US findings. In this case, a second confirmatory benign FNAC should be obtained.

\section{Indeterminate lesions (TIR 3)}

Management of indeterminate thyroid nodules should be not only based on their cytologic sub-classification, but also on clinical data, US findings, and, possibly, mutational analysis. The use of molecular tests, however, is still expensive and should be restricted to specialized centers for selected patients. Interdisciplinary consultation is recommended in the management of these cases, and the diagnostic and therapeutic options should be discussed with the patient.

\section{Low-risk indeterminate lesions (TIR 3A)}

The expected risk of malignancy is $5-15 \%$. Close follow-up is suggested as the preferential option in most cases. Conservative management is supported by favorable clinical criteria, based on personal and family history and small size of the lesion, but the most important factor in decision making is represented by low-risk US features. A repeat FNA reviewed by an experienced cytopathologist is recommended, but may still not offer conclusive information. Patients with suspicious clinical or US findings should preferentially be treated with lobectomy. Total thyroidectomy may be considered depending on the clinical setting, coexistence of contralateral nodules and patient preference.

\section{High-risk indeterminate lesions (TIR 3B)}

The expected risk of malignancy is $15-30 \%$. Repeat FNA of nodules classified as follicular neoplasm is not generally recommended, because it does not provide additional information for management. Surgical excision of the lesion with histologic examination should be performed in most cases. In patients with favorable clinical and US features, a close clinical follow-up without immediate diagnostic surgery may considered.

Patients are preferentially treated with lobectomy. Frozen sections are usually not recommended, but may be useful to decrease the risk of subsequent completion thyroidectomy in the scenario of cancer diagnosis. Total thyroidectomy may be performed on the basis of the clinical setting, coexistence of contralateral thyroid nodules, and patient preference.

\section{Suspicious nodules (TIR 4)}

The rate of histologically confirmed malignancy in these cases is about $60-75 \%$, papillary carcinoma being the most frequent histologic type. Indications for surgery are quite similar to nodules with TIR 5 cytology.

\section{Malignant nodules (TIR 5)}

This category includes cases with a conclusive cytologic diagnosis of malignant neoplasm. TIR 5 cytological diagnosis accounts for $2.7-5 \%$ of the FNA, with a risk of malignancy greater than $98 \%$. Treatment options should be discussed with the patient. Surgical excision should be recommended and its potential complications discussed. The surgical approach and its extent should be planned according to the clinical setting and the imaging findings. For large tumors with pre-surgical evidence of local metastases, total thyroidectomy and removal of lymph nodes are indicated. For patients with thyroid cancer $>1$ and $<4 \mathrm{~cm}$ without extra-thyroidal extension or clinical evidence of lymph node metastases, the initial surgical procedure can be either total thyroidectomy or lobectomy, depending on the willingness of the specialist and the patient to perform postsurgical radioiodine ablation. A close clinical follow-up may be offered to patients with very low-risk tumors (papillary microcarcinomas with no clinical evidence of extra thyroid spread or metastases) and to elderly patients with incidentally discovered papillary cancer, who are at high surgical risk and have no evidence of extra thyroid spreading.

\section{Item 3: What is the role of pre-operative staging with diagnostic imaging and laboratory tests?}

\section{Recommendation statement}

(a) Accurate pre-operative staging of DTC is based on neck US and it is mandatory in all patients to carry out the most appropriate surgical treatment.

(b) Suspicious neck lymph nodes should be submitted to FNAC complemented with thyroglobulin measurement in the needle washout (FNA-Tg).

(c) In patients presenting with US and/or clinical evidence/ suspicion of locally advanced thyroid carcinoma, crosssectional imaging studies (e.g., CT and/or MR) are recommended for accurate surgical planning. 
(d) Routine serum $\mathrm{Tg} / \mathrm{Tg} \mathrm{Ab}$ measurement before surgery is not recommended.

(e) Serum calcitonin (CT) measurement before surgery is recommended.

\section{Narrative of the recommendation}

Lymph node metastases occur quite frequently in patients with DTC; therefore, pre-surgical staging has a strategic role to optimize surgical cure [1,5-8]. Moreover, in patients with clinically low-risk DTC, the information obtained through the pre-surgical staging may be critical to define the most appropriate treatment option, which may encompass active surveillance as well as conservative surgery (i.e., lobectomy) or total thyroidectomy with or without central neck dissection.

US is the more sensitive imaging technique for the examination of the thyroid gland as well as the neck lymph nodes, thus playing a pivotal role in the pre-operative staging of patients candidate to surgical treatment of DTC. US resolution in detecting minimal extrathyroidal extension of the tumor, as well as microscopic neoplastic foci is less than optimal. In addition, US sensitivity in detecting lymph node metastases in the central compartment prior to thyroidectomy is quite low. Notwithstanding these limitations, USbased staging of the disease should aim to provide the following information:

- site and size of the primary tumor and of suspicious lymph nodes;

- evidence of tumor multifocality/bilaterality and of extrathyroidal spreading;

- other neck masses and gross vascular abnormalities.

The above-mentioned US signs and patterns which suggest malignancy of neck lymph nodes do not have absolute specificity. The measurement of thyroglobulin on needle washout (FNA-Tg) is a valuable diagnostic aid, reducing the risk of non-diagnostic results due to cytological samples of poor quality. FNA-Tg levels in metastatic thyroid lymph nodes are usually exceedingly high (>500-1000 ng/ $\mathrm{mL}$ ), and allow a clear-cut diagnosis; nevertheless, FNA-Tg results should always be weighed against the levels of circulating serum $\mathrm{Tg}$. In most cases, the presence of serum anti$\mathrm{Tg}$ antibodies does not interfere with FNA-Tg measurement.

In a minority of cases, DTC may present with signs and symptoms suggesting invasion of deep neck structures and/ or mediastinum, which cannot be adequately visualized by neck US. Cross-sectional imaging of the neck, mediastinum, and lung is required in the following settings:

- primary tumor and/or loco-regional metastases only partially imaged by neck US;
- voice changes, dysphagia, dyspnea or other symptoms of mediastinal extension;

- rapidly enlarging palpable and firm neck masses.

When CT scan with iodine-containing contrast medium is performed, a 1 month-interval between CT study and ${ }^{131} \mathrm{I}$ administration is required. Patients presenting with signs and symptoms suggesting tumor invasion into the airways and/ or the digestive tract should undergo tracheobronchoscopy and/or esophagoscopy. Screening for other sites of distant metastases is not recommended on routine bases.

The measurement of serum $\mathrm{Tg}$ and $\mathrm{TgAb}$ levels prior to thyroidectomy does not provide any useful information for disease staging. Increased serum $\mathrm{Tg}$ levels (when present) reflect the size of the normal thyroid and of the nodule(s) and the functional thyroid status rather than the nature of the nodule(s).

Serum calcitonin (CT) measurement is useful to reveal medullary thyroid cancer. Not all authors recommend calcitonin measurement in all the nodules; thus, a good compromise may be to advocate $\mathrm{CT}$ measurement at least in patients undergoing surgery for suspicious cancer or in patients with multinodular goiter.

\section{Item 4: When thyroidectomy is indicated which strategy should be performed: total or lobo-isthmectomy?}

\section{Recommendation statement}

(a) Thyroid lobo-isthmectomy alone is an adequate initial surgical treatment for patients with suspicious cancer $\leq 1 \mathrm{~cm}$, clinically limited to one lobe, with no evidence of extrathyroidal extension or metastatic disease to nodes (cNOb) or prior head and neck irradiation.

(b) Total thyroidectomy is recommended with at least one of the following parameters: patients with differentiated thyroid carcinoma $>4 \mathrm{~cm}$, unilateral or multifocal disease, with at least one of the following: clinically (or intraoperative) detected cervical nodal metastases, gross extrathyroidal extension or metastatic disease to distant sites.

(c) Lobo-isthmectomy or total thyroidectomy may be proposed to patients with differentiated thyroid carcinoma $>1$ and $\leq 4 \mathrm{~cm}$ without clinical (or intraoperative) evidence of extrathyroidal extension and lymph node metastases (NOb). Total thyroidectomy may be preferred to enable radioiodine treatment and to enhance follow-up accuracy, or for preference of the patient. 


\section{Narrative of the recommendation}

Extension of thyroidectomy for patients with differentiated thyroid carcinoma is still matter of debate. Many guidelines have supported total thyroidectomy as the primary initial surgical treatment option for nearly all differentiated thyroid carcinomas greater than $1 \mathrm{~cm}$ with or without evidence of nodal or distant metastases. This approach was based on retrospective data suggesting that bilateral surgical procedure would decrease recurrence rates [9]. Recent data have demonstrated that in properly selected low-risk patients, the overall survival and cause specific survival rates are similar following unilateral or bilateral surgery $[10,11]$.

Considering recent evidence patients and surgeons should carefully balance the relative benefits and risks of total thyroidectomy vs. thyroid lobo-isthmectomy. Most of these cancers are not aggressive and could be treated with a unilateral procedure only, because the percentage of complications for total thyroidectomy is not irrelevant, while clinical surveillance and the possibility of second step surgery can reassure both surgeon and patient about the final outcome. This choice should include surgeon volume (that is, referral to dedicated thyroid surgeons) due to the relationship between high-volume thyroid surgical center and patient outcomes

\section{Item 5: No intervention for papillary thyroid microcarcinoma}

\section{Recommendation statement}

(a) Even if surgery is the treatment of choice, "no immediate intervention" and active surveillance may be considered for very low-risk PTMC in the following setting:

1. patients at high surgical risk;

2. patients who refuse surgical treatment;

3. patients willing to enter into controlled clinical trials.

(b) A personal decision making is recommended as well as an accurate discussion with the patient to explain pro and cons of the active surveillance vs. surgical treatment.

(c) A careful clinical and cytological evaluation of risk factors for aggressive behavior or recurrence of PTMC is recommended. A repeated "neck" sonographic evaluation after cytological suspicion of PTMC should be the first step to be carried out to exclude the presence of suspicious lymph nodes.

\section{Narrative of the recommendation}

PTMC has an excellent prognosis and its natural history displays a very low progression [12]. However, since a minority of observed PMTC over time showed increases tumor size or the appearance of lymph node metastases, a long-term active surveillance is recommended.

Clinical patient history, physical and US examination, and molecular investigation cannot really distinguish the small subgroup of PTMC at risk of developing local or distant metastases from the majority of PTMC associated with an indolent course.

In case of choice of "active surveillance" of PTMC, neck ultrasound should be repeated every 6 months in the first 2 years and once a year thereafter: a significant increase in size of the nodule or the evidence a suspicious lymph node not present at the previous controls are the main reasons to re-thing the therapeutic strategy.

Risk factors for aggressive behavior and recurrence of PTMC include: previous neck irradiation; extra-thyroidal extension at US; subcapsular or posterior localization of PTMC; multifocal or bilateral tumor; coexistence of Graves' disease; suspicious lymph node involvement; aggressive cytological features; and BRAF mutation (if available).

\section{Item 6: Indication for completion thyroidectomy after lobectomy}

\section{Recommendation statement}

(a) Completion thyroidectomy is indicated to those patients for whom a total thyroidectomy would be suggested as the initial surgical treatment in case of primary diagnosis of differentiated thyroid cancer.

(b) Completion thyroidectomy is mandatory for differentiated thyroid carcinoma $>4 \mathrm{~cm}$, or any differentiated thyroid carcinoma with extrathyroidal extension and/or with histological evidence of lymph node metastases or aggressive variants.

(c) In the other cases, completion thyroidectomy is not routinely indicated, but the final decision should be taken based on a tailored strategy involving a careful discussion with the patient.

Completion thyroidectomy is not indicated for a final histology of NIFT-P [13].

\section{Narrative of the recommendation}

Since differentiated thyroid carcinomas can be managed with either lobo-isthmectomy or total thyroidectomy, a completion thyroidectomy is not always required. 
Every case is crucial to weigh the relative advantages and disadvantages of thyroid lobectomy with completion thyroidectomy vs. initial total thyroidectomy. Risk group stratification and post-operative risk-adapted evaluation is mandatory. The multidisciplinary team may choose the treatment of choice evaluating also patient's preferences and follow-up opportunities.

The surgical risks of two-stage thyroidectomy are similar to those for a total thyroidectomy, but this is true only in specialized centers [14]. The accuracy of the first surgery is crucial to minimize the risk of re-operation. In particular, reoperation is safer in the presence of not dissected contralateral thyroid lobe when thyroid lobectomy was carried out en bloc with thyroid isthmus. Nonetheless, the evidence of inferior laryngeal nerve palsy following thyroid lobectomy calls for strong caution in deciding to perform completion thyroidectomy.

Patients with low-risk tumors must undergo surveillance imaging to detect remnant gland recurrence. In experienced hands, ultrasound is the tool of choice to follow-up these patients, ensuring an adequate diagnostic accuracy. Thus, patients can be treated with completion thyroidectomy if necessary at a later date, avoiding unnecessary surgery and limiting the risk of post-operative complications.

\section{Item 7: The role of central and lateral compartment neck dissection in the management of DTC}

\section{Recommendation statement}

(a) Prophylactic central compartment neck dissection (CCND) is not routinely indicated.

(b) CCND is appropriate for patients with differentiated thyroid carcinomas in the presence of clinically involved lymph nodes (cN1) either in the central compartment or in the lateral neck.

(c) Lateral neck dissection is appropriate for patients with differentiated thyroid carcinomas in the presence of metastatic lymph nodes $(\mathrm{cN} 1)$ in the lateral neck.

(d) Intraoperative surgeon judgement is critical to decision making on whether central neck dissection is to be performed.

\section{Narrative of the recommendation}

Given the high incidence of occult nodal metastases in papillary thyroid carcinoma (PTC), prophylactic CCND has been proposed as the initial management of these tumors. Nevertheless, the role of prophylactic CCND in the management of thyroid cancer remains controversial [15-17].

The potential benefits of include:
- eliminating a possible source of recurrence and preventing the morbidity of revision surgery;

- increasing the accuracy of disease staging for radioactive iodine therapy;

- improving the accuracy of thyroglobulin surveillance and long-term follow-up.

While occult node positivity is quite common in PTC, these occult metastases tend to be small in size and number, with no extranodal extension, and a median risk of recurrence of only $2 \%$ at 5 years. The comparability in oncologic outcome between patients who undergo prophylactic CCND and those who do not confirms the indolent biologic behavior of subclinical nodal disease [17]. In addition, meta-analyses seem to show that the presence of occult central nodal metastases is not a significant predictor of recurrence nor did prophylactic CCND offer improved local control [18].

On the other hand, it should be considered that CCND increases the risk of hypoparathyroidism and, with a lower degree of evidence, of recurrent nerve palsy when compared to total thyroidectomy alone.

Prophylactic ipsilateral central compartment neck dissection, including pre-laryngeal, pretracheal, and paratracheal nodal basins on the side of the tumor, with frozen section examination, could be proposed in selected patients [19].

\section{Item 8: The need for a complete histological and cytological report}

\section{Recommendation statement}

\section{Histology (see Appendix 1):}

(a) The thyroid specimen should accurately describe the weight (or the measurements of the lobes), the characteristics of the nodule(s), and the presence of periglandular fat.

(b) The histological report should include the predominant histotype and, if present, only a minor component of either an aggressive variant (diffuse sclerosing variant, tall/columnar cell, hobnail, solid) or a less differentiated tumor type (insular, trabecular, and anaplastic).

(c) The histology should also report the presence of capsular (thyroid and tumor) invasion, of vascular infiltration (no. of involved vessels), additional neoplastic foci (size and site) and lymph nodes (eventual micro-metastases $<2 \mathrm{~mm}$ and size of the larger node).

(d) Immunohistochemical and molecular analyses, when performed, should be reported or there should be a reference to an additional report (e.g., 
molecular studies). TNM report should follow the VIII edition of AJCC/UICC [20].

2. Cytology:

(a) The cytological report should specify the type of technique used to obtain the sample (FNAC, with or without US guide or other) and the size of the needle used (Gauge).

(b) Essential clinical information must be included with the sample such as the structure of lesion (cystic vs. solid), the location (isthmus vs. lobes), the number of lesions analyzed and their size, the functional status, and the sonographic pattern (see "Item 1" and ATA guidelines).

(c) Indicate the method used to process the sample (conventional smear vs. liquid based cytology).

(d) Use the Italian reporting system to classify the lesions:

TIR 1 non-diagnostic (risk of malignancy not defined).

TIR 1C cystic $(<10 \%)$.

TIR 2 negative for malignant cells/benign $(<3 \%)$.

TIR 3A low-risk indeterminate lesion (5-10\%).

TIR 3B high-risk indeterminate lesion (20-30\%).

TIR 4 suspicious of malignancy (60-80\%)

TIR 5 positive for malignant cells ( $>95 \%)$.

\section{Narrative of the recommendation}

Aggressive variants of papillary carcinoma.

The variants with more unfavorable outcomes are the tall cell, columnar cell, and hobnail variants. Their knowledge may contribute to risk stratification. At least $30 \%$ of "tall cell", according to Ganly et al. [21] or 10\%, according to Dettmer et al. [22], should be present to diagnose the tall cell variant. This variant is associated with higher rate of lymph node metastasis and poorer survival independently of patient age, tumor size and stage [23]. The columnar cell variant is characterized by predominance of columnar cells with pronounced nuclear stratification. These tumors have a higher risk of distant metastases and tumor-related mortality.

Papillary carcinoma with prominent hobnail features is a rare, recently described variant characterized by the predominance of cells with a hobnail appearance with apically placed nuclei and bulging of the apical cell surface [24]. It is associated with frequent distant metastases and increased risk of tumor-related death.

Other variants of papillary carcinoma, such as the solid variant and diffuse sclerosing variant, have been traditionally associated with a less favorable outcome, although the data remain conflicting.
The solid variant is more frequently associated with distant metastases that are present in about $15 \%$ of cases, and with a slightly higher mortality rate, which was $10-12 \%$ in two studies with 10 and 19 years of average follow-up [25]. The solid variant of papillary carcinoma should be distinguished from poorly differentiated thyroid carcinoma, with which it shares the insular, solid, and trabecular growth patterns. The distinction is based primarily on the preservation of nuclear features and lack of necrosis and high mitotic activity in the solid variant, as outlined by the Turin diagnostic criteria for poorly differentiated thyroid carcinoma [26, 27].

The prognostic implication of the diffuse sclerosing variant of papillary cancer remains controversial. This variant is characterized by diffuse involvement of the thyroid gland and it has lower disease-free survival [28] and a higher rate of local and distant metastases at presentation [29].

\section{Lymph nodes}

The number of involved LNs, the size of the largest metastatic focus within the lymph node, the location of the lymph nodes, and the presence of extranodal extension are all determinant of the risk of recurrence. Sugitani et al. [30] demonstrated that risk of recurrence at 10 years following total thyroidectomy and neck dissection without RAI ablation was significantly higher in patients with $\mathrm{pN} 1$ disease with the largest metastatic $\mathrm{LN}>3 \mathrm{~cm}$ (27\%) than in patients with $\mathrm{pN} 1$ disease $<3 \mathrm{~cm}(11 \%)$. The risk of recurrence was significantly higher in patients with $>5 \mathrm{LN}$ metastases (19\%) that in those with $<5 \mathrm{LN}$ metastases (8\%). Consideration should also be given to reporting the specific histologic features of the LN metastases (e.g., specific histologic variant and the presence of tumor necrosis/mitosis). Based on these features, it has been proposed to differentiate lower risk N1 disease (Clinically N0, Micro-metastases, or less than five small lymph nodes, with $<5 \%$ risk of recurrence) from higher risk $\mathrm{N} 1$ disease $(\mathrm{cN} 1$, lymph nodes $>3 \mathrm{~cm}$ or $>5$ metastatic nodes, with $>20 \%$ risk of recurrence).

\section{Item 9: Post-surgical thyroid ablation with radioiodine: routine or selective indication}

\section{Recommendation statement}

(a) The indication to post-surgical thyroid ablation with radioiodine should be established both on the basis of the AJCC/UICC staging (VIII edition) and the Initial Risk Stratification System proposed by ATA, useful to predict the risk of mortality and of disease recurrence/ persistence, respectively.

(b) The indication should also be based on the post-operative disease status evaluated by serum Tg (on thy- 
roid hormone therapy or after TSH stimulation) and neck US. In selected cases, other imaging procedures (including diagnostic RAI WBS) may be indicated.

(c) In patients with ATA low-risk (T1a-b, N0-X, M0-X), RAI remnant ablation is not generally recommended. However, consideration of specific features could lead to consider RAI remnant ablation in individual patients.

(d) In patients with intermediate or low-to-intermediate risk (T1-2, N1a-N1b, M0-X), RAI remnant ablation should be considered, particularly in patients with adverse features such as advanced age, larger tumors, macroscopic or clinically evident lymph nodes or the presence of extranodal extension, or aggressive histology or vascular invasion.

(e) In patients with high risk or intermediate to high risk (T3-4, any N, any M), RAI ablation is routinely recommended.

\section{Narrative of the recommendation}

When deciding whether to perform or not thyroid ablation, two concept should be taken into account:

- Even if it has been decided not to perform RAI ablation immediately after surgery, it can be done at any time during the follow-up if the opportunity/necessity comes up.

- Post-operative serum Tg and neck US have been demonstrated to be useful in predicting the result of 131-I WBS and when basal serum Tg or TSH-stimulated Tg are undetectable or $<5 \mathrm{ng} / \mathrm{mL}$, respectively, associated with a negative neck US the probability to have local or distant metastases is negligible and thus remnant ablation can be avoided.

Our statement is based on the following consideration:

- In low-risk DTC patients, there is little evidence to suggest that thyroid ablation may improve the very low risk of disease-specific mortality and to have an effect on the low rate of recurrence [31-33]. Besides, it is not demonstrated that delayed diagnosis and treatment of persistent disease may decrease the chance of cure. Retrospective multicenter studies and prospective data did not show significant effect of thyroid ablation on overall or disease-free survival, both using multivariate and stratified propensity analysis. In systematic reviews, the majority of studies did not show a significant effect of thyroid ablation in reducing thyroid cancer-related death, and conflicting findings have been provided regarding disease recurrence. The majority of the studies suggest that RAI adjuvant therapy is unlikely to improve disease-specific or disease-free survival in unior multifocal papillary microcarcinoma, in the absence of other higher risk features. However, only the results from prospective randomized controlled trials, at moment in progress, should definitely indicate which patients with low-risk DTC may or may not benefit from post-operative RAI.

- For intermediate-risk DTC, the greatest potential benefit (improved overall survival) of the thyroid ablation are observed in patients with aggressive papillary thyroid cancer histologies, primary tumor $>4 \mathrm{~cm}$, evidence of extrathyroidal extension, increasing volume of nodal disease, lymph node disease outside the central neck (N1b disease), lymph nodes $>1 \mathrm{~cm}$ in diameter and advancing patient age ( $>45$ years) [34]. A systematic review evaluating the benefit of RAI in intermediate-risk patients, reported a significant benefit of RAI treatment in improving overall and disease-specific survival as well as disease-free survival in about half of the non-randomized studies. However, more research is needed to understand the therapeutic efficacy of RAI in various subgroups of patients in the intermediate-risk category.

- Data from prospective multicenter studies on papillary or follicular high-risk DTC patients suggest that post-surgical RAI therapy is associated with improved overall survival and disease-specific mortality. The overall survival in FTC patients with iodine-positive distant metastases is more than doubled in patients receiving post-surgical RAI treatment.

\section{Item 10: Preparation for thyroid ablation and selection of the most appropriate activity}

\section{Recommendation statement}

\section{Preparation for radioiodine ablation}

(a) In patients with low and intermediate ATA risk DTC without extensive lymph node involvement, preparation with recombinant human TSH (rhTSH) stimulation is equally effective compared to thyroid hormone withdrawal (THW) but with the advantage of preserving the quality of life.

(b) In patients with ATA high-risk DTC and high risk of disease-related mortality and morbidity, more controlled data from long-term outcome studies are needed to recommend the routine use of rhTSH

(c) In patients with DTC of any risk level presenting with significant medical or psychiatric comorbidity who cannot tolerate hypothyroidism or are unable to obtain an elevation of TSH by THW, rhTSH preparation should be considered.

(d) In preparation for thyroid ablation, avoidance of iodinecontaining drugs or iodinated contrast agents is manda- 
tory. A low iodine diet avoiding preserved fish, sushi, seaweed for 1-2 weeks should be recommended

(e) Except for patients with suspected iodine contamination, the routine measurement of urine iodine excretion before RAI thyroid remnant ablation may not be necessary.

\section{Selection of the most appropriate activity of radioiodine for remnant ablation}

(a) In patients with low-risk or intermediate-risk thyroid cancer, a low administered activity of radioiodine (30$50 \mathrm{mCi}$ ) for remnant ablation is generally favored being low activities as effective as high activities in obtaining complete ablation. Higher administered activities (100 mCi or more) should be considered for patients at high risk of persistent/recurrent disease, when the administration is intended in terms of adjuvant therapy or for patients submitted to less than a total or near-total thyroidectomy.

\section{Narrative of the recommendation}

A precondition to the radioiodine administration for thyroid remnant ablation is an adequate elevation of serum thyroidstimulating hormone (TSH). Serum TSH elevation may be obtained using two methods: (a) thyroid hormone withdrawal and (b) administration of exogenous rhTSH.

(a) If thyroid hormone withdrawal is planned, levothyroxine (LT4) should be withdrawn for 3-4 weeks. If $\mathrm{LT}_{4}$ is withdrawn for 6 weeks, triiodothyronine (LT3) may be substituted for LT4 in the initial 4 weeks, and withdrawn in the last 2 weeks. Serum TSH should be measured prior to radioiodine administration to verify the appropriated degree of TSH elevation.

(b) When using rhTSH, the recommended dosage is $0.9 \mathrm{mg}$ given by two i.m. injections to the buttock in the 2 days preceding the radioiodine administration. Routine measurement of serum TSH levels is not recommended. rhTSH is currently approved in patients who have undergone a near-total or total thyroidectomy for well-differentiated thyroid cancer and who do not have evidence of distant metastases [35]. Remnant ablation after preparation with rhTSH is associated with superior short-term quality of life, reduced whole-body irradiation and chromosomal abnormalities and similar rates of successful remnant ablation as compared to traditional hypothyroidism. Although long-term outcome data following rhTSH preparation for RAI treatment are limited, the two studies published so far clearly show the same outcome of the patients independently from the type of stimulation. However, these studies are limited to low- and indeterminate-risk cases and randomized control trials are needed to guide clinical care in higher risk DTC patients.

A systematic reviews/meta-analyses of the studies show that similar rates of successful remnant ablation can be achieved using activities of 131I between 30 and $100 \mathrm{mCi}$ in adult patients with well-differentiated thyroid carcinoma who had been treated with total or near-total thyroidectomy and who were not known to have any gross residual disease following surgery. No difference in terms of ablation success rates was found in two prospective randomized studies conducted in France [36] and in the United Kingdom [37] using 30 or $100 \mathrm{mCi}$ of $131 \mathrm{I}$ after preparation with thyroid hormone withdrawal or rhTSH. Regarding the impact of the low RAI activities for remnant ablation on long-term outcome, more controlled data from long-term studies are needed [38-40].

1. In preparation for remnant ablation, many centers recommend the use of a low-iodine diet (LID) to reduce the influence of the stable iodine on the uptake of therapeutic activities of radioiodine. A recent systematic review of observational studies shows a reduction in urinary iodine excretion as well as an increase in 131I uptake in patients prepared with the most commonly studied LID when compared with patients not prepared with LID. On the other hand, a lack of association between urine iodine excretion and rate of successful thyroid ablation has been reported in patients not specifically prescribed an LID, suggesting that the routine measurement of urinary iodine excretion may not be necessary. A retrospective study [41] shows no influence of different levels of urinary iodine on the outcome of thyroid ablation up to urinary iodine levels exceeding $350 \mu \mathrm{g}$ /day of dietary iodine. In addition, occasionally, even patients on LID may show urinary iodine levels exceeding 500-1000 $\mu \mathrm{g} /$ day. The use of iodized salt should not be restricted.

\section{Item 11: Informing the patient treated with radioiodine on safety measures}

References [42-44].

\section{Recommendation statement}

(a) Radioiodine treatment of thyroid cancer must be performed in protected hospitalization with collection of patient excreta.

(b) At discharge, written information and instructions of behavior must be provided to the patient and his family 
to respect the dose limits for the population and dose constraints for the family members or caregivers.

(c) Particularly, the written information at discharge must concern the patient travel after discharge, behavior in public and domestic environments, return to work, pregnancy and radiation-detection systems at international borders, airports, and other public areas.

(d) Pregnancy is an absolute contraindications to 131I therapy. Pregnancy should be delayed for 6-12 months after radioiodine therapy; in men, conception should be avoided for 4 months after 131I therapy.

(e) Women who are lactating or have recently stopped breastfeeding should not be treated with 131I. Breastfeeding must be stopped at least 6 weeks before administration of 131I therapy.

\section{Narrative of the recommendation}

The obligation of treatment in protected hospitalization with collection of patient excreta results by the Italian law 187/2000, transposition of the European directive 97/43 MED.

Witten instructions are provided to the patient and his family at discharge to respect the dose limits for the population and dose constraints for the family members or caregivers. After radioiodine administration, the main source of radiation dose to family members, caregivers, and the general public comes from external exposure to high-energy gamma rays, according to three variables: the retained radioiodine activity, the distance (inversely proportional to the square of the distance from the patient) and the duration of exposure (directly proportional to the time spent close to the patient). The second potential radiation exposure pathway of family members or caregivers is the contamination through 131I excreted/secreted by the treated patient. The majority of the excretion of radioiodine occurs via the urine, maximal during the first $48 \mathrm{~h}$ after treatment. Small amounts of 131I concentrations are present in the saliva for as long as 7 days, stool, and other body fluids. Although contamination is much less important than external exposure as source of radiation dose, it is very important to pay attention to young children whose thyroid glands and other tissue are more sensitive to radiation.

Pregnancy is an absolute contraindications to 131I therapy because of the consequent fetal irradiation and the possible transfer of radioiodine to the fetal thyroid. After radioiodine treatment, pregnancy should be delayed for at least 6 months.

During lactation, the mammary glands concentrate iodide at about 30 times the free inorganic plasma iodide concentrations via the increased expression of sodium iodide symporter (NIS). Therefore, breastfeeding should be discontinued to prevent $131 \mathrm{I}$ in the milk from reaching the infant's thyroid gland and to limit the radiation absorbed dose to the mother's breast tissue.

In young men, radioiodine therapy has been associated with a temporary reduction in sperm counts and transient elevated serum follicle-stimulating hormone (FSH) levels. However, for patients treated with a single ablation dose testicular function recovers within few months and the risk of infertility is almost absent. Permanent testicular damage may be observed after high cumulative doses of radioiodine. Sperm banking could be considered in young patients with distant metastases likely requiring high cumulative activities.

Sensitive radiation detectors installed in airports, public transportation facilities, and other ports of entry can be triggered by patients who have received radioiodine. It is possible that patients treated with 131I could trigger alarms at such detection sites for 95 days or longer after treatment. If, after receiving 131I therapy, travel across international borders or via airports is planned, a form including the date of treatment, the radionuclide, the administered activity and the treating facility should be provided to the patient.

\section{Item 12: Follow-up after initial treatment (surgery and radioiodine): when and how}

\section{Recommendation statement}

(a) The initial follow-up visit, aimed at defining the response to primary treatment, should be performed at 6-12 months.

(b) In patients who have had total thyroidectomy with RAI remnant ablation or adjuvant therapy, the first-level tools for assessing responses to initial treatment should be: (a) serum $\mathrm{Tg}$ measurement on L-thyroxine therapy with a sensitive Tg assay or after TSH stimulation; (b) serum $\mathrm{Tg}$ antibodies measurement (not the recovery test); and (c) neck ultrasound.

(c) Functional (whole-body RAI scan, 18-fluoro-2-deoxyglucose positron emission tomography scan) and/or cross-sectional (computed tomography, magnetic resonance imaging) studies may be considered in patients with high risk of persistent disease, when response to therapy cannot be correctly classified with first-level tools.

(d) In patients with neck lesions sonographically suspicious for malignancy, FNA cytology with Tg assay in the needle-washout fluid is recommended if this would change management. 


\section{Narrative of the recommendation}

The goals of the early post-operative follow-up are to tailor the TSH level to each patient's risk of recurrence (2-3 months after initial treatment) and to identify patients who are disease-free and can be safely managed with less intensive surveillance, and those with persistent disease, who may require additional therapy or a more active surveillance (6-12 months after initial treatment) [1]. Serum $\mathrm{Tg}$ assays and neck ultrasound are regarded as the first-level tools for assessing responses to initial treatment $[2,45,46]$. Functional and cross-sectional imaging studies play secondline roles.

Undetectable serum ultrasensitive Tg levels (either basal or rhTSH-stimulated) and negative $\mathrm{TgAb}$ can reliably identify patients who are disease-free, with a negative predictive value close to $100 \%$. Minimally detectable Tg levels, especially after TSH stimulation, carry a very low positive predictive value. Indeed, most patients with incomplete or indeterminate response to therapy remain free of structural disease during prolonged follow-up. Reliable Tg cutoffs for distinguishing the meaning of detectable serum $\mathrm{Tg}$ have not been established. This reduces the specificity of positive $\mathrm{Tg}$ assay, especially when RAI remnant ablation is omitted. Therefore, $\mathrm{Tg}$ trends rather than absolute levels should be monitored [47]. Declining levels are reassuring, whereas rising values suggest the presence of growing thyroid tissue or cancer. However, on this regard, it is worth to note that to compare Tg values, the measurement should be done possibly in the same laboratory and with the same assay. The TSH values must also be considered, since small variations of TSH can greatly affect the serum Tg values. Immunometrically determined $\mathrm{Tg}$ levels can be falsely lowered by the presence of Tg autoantibodies which must always be measured simultaneously to Tg. Temporal trends in the antibody titers can help to distinguish residual normal thyroid tissue from recurrent tumor, although they are less reliable than serum $\mathrm{Tg}$ trends.

Cervical ultrasound provides prompt information during follow-up, including the initial phase when $\mathrm{Tg}$ assay results may be difficult to interpret. Thyroid cancer spread or recurrence usually begins in the cervical lymph nodes, where it can be sonographically identified using well-established criteria. Ultrasound-guided FNA and FNA-Tg can be used to confirm suspicious findings thereby increasing specificity (see "Item 3"). In patients at high risk of persistent disease, or in the presence of negative findings on neck US associated with elevated serum $\mathrm{Tg}$ levels or rising serum $\mathrm{Tg}$ or $\mathrm{Tg}$ antibody levels, second-line imaging studies should be used to exclude the presence of metastatic lesions.
Item 13: Risk stratification incorporating the response to initial treatment

\section{Recommendation}

(a) The ATA Initial Risk Stratification System (low-intermediate-high risks) is recommended for DTC patients treated with thyroidectomy.

(b) At 6-12 months, the initial recurrence risk estimates should be modified to incorporate the response to initial therapy.

(c) Risk estimates should be further modified during follow-up, as a function of the clinical course of the disease.

\section{Narrative of the recommendation}

Initial risk stratification provides important information into an individual patient's risk of recurrence and disease-specific mortality and can dictate the need for radioiodine therapy. However, they are derived only from personal and pathological features available at the time of initial therapy, and thus, they do not take into account the response to initial therapy or to new features discovered during follow-up. By incorporating this additional information, we can get a dynamic risk stratification which can change over time according to the clinical course of the disease. An ATA low-risk patient that develops cervical lymphadenopathy at some point during follow-up would be reclassified as intermediate or high risk. Conversely, an ATA high-risk patient that has no evidence of disease during follow-up would be reclassified as low risk. Dynamic risk stratification avoids aggressive treatment for patients that after initial therapy become free of disease (low risk) regardless of the initial risk attributed based on personal data and histopathology.

Response to initial therapy includes the following categories:

1. Excellent response: negative or non-specific structural or functional imaging findings and either suppressed $\mathrm{Tg}<0.2 \mathrm{ng} / \mathrm{mL}$ or TSH-stimulated $\mathrm{Tg}<1 \mathrm{ng} / \mathrm{mL}$;

2. Biochemical incomplete response: negative imaging and detectable basal or stimulated $\mathrm{Tg}$ or rising anti-Tg antibody levels;

3. Structural incomplete response: evidence of disease at structural or functional imaging, with any Tg level, with or without anti-Tg antibodies. 


\section{Item 14: Follow-up of patients with excellent response to initial therapy}

\section{Recommendation statement}

(a) In DTC patients with an excellent response to initial treatment, follow-up should be performed every 12-24 months.

(b) In patients who achieve an excellent response to therapy, irrespective of initial treatment, follow-up should be based on measurement of basal Tg levels (with ultrasensitive assays $<0.2 \mathrm{ng} / \mathrm{mL}$ ) under L-thyroxine, anti thyroglobulin antibodies and neck ultrasound.

(c) In these patients, L-thyroxine therapy should aim to keep TSH levels in the low-normal range (0.5-2 mUI/L).

\section{Narrative of the recommendation}

Patients' follow-up should be performed every 12-24 months. After 5 years, the frequency of follow-up visit may be lengthened. As tumor recurrence can happen also several years after surgery/radio remnant ablation, follow-up should be continued lifelong [48].

In patients who underwent total thyroidectomy (without RAI), there are not specific cutoff to assess the absence of tumor persistence/relapse because of the possible presence of the normal thyroidal tissue. Monitoring Tg levels to assess the Tg trend, together with neck US, is suggested to distinguish between residual normal thyroid tissue and tumor relapse [47].

In patients who underwent successful RAI remnant ablation after surgery, undetectable ultrasensitive serum $\mathrm{Tg}$ levels $(<0.2 \mathrm{ng} / \mathrm{mL})$ on LT4 are highly predictive of tumor absence. Rising Tg values overtime are highly predictive of recurrent disease [49].

In addition, as most of DTC recurrences occur in the neck (in thyroid bed or in cervical lymph nodes), combining neck US to Tg assessment is the most accurate follow-up strategy for these patients [50]. Neck US can detect structural tumor relapse also in the absence of Tg elevation. In case of suspicious neck lesions, US guided FNA should be considered, with $\mathrm{Tg}$ assay on needle-washout fluid.

In case of undetectable Tg on LT4 and in the absence of suspicious findings, further Tg measurement after TSH stimulation is not routinely recommended.

\section{Item 15: Follow-up of patients with persistent structural disease or biochemical disease}

\section{Recommendation statement}

\section{Persistent biochemical disease}

(a) Patients with persistent biochemical disease should undergo periodic $\mathrm{Tg}$ and $\mathrm{TgAb}$ measurements and neck ultrasound on 1-T4 treatment, approximately every 6-12 months.

(b) A progressive increase of $\mathrm{Tg}$ level with a doubling time period lower than 12 months indicates a rapid progression and requires additional morphological examinations to assess the presence of structural disease.

(c) TSH-stimulated $\mathrm{Tg}$ testing may be helpful only for re-staging patients, after additional therapies. In these patients, we recommend a moderate (TSH 0.1$0.5 \mathrm{mIU} / \mathrm{mL}$ ) or complete (TSH $<0.1 \mathrm{mIU} / \mathrm{mL}$ ) TSH suppression that should be individualized according to $\mathrm{Tg}$ or $\mathrm{TgAb}$ values and their trend of increase.

\section{Persistent structural disease}

(a) Patients with persistent structural disease need an active surveillance and should undergo periodic morphological examinations to assess the status of disease and to evaluate the need and the appropriateness of treatment.

(b) An individualized follow-up program should be planned according to site and burden of metastases, 131I uptake, and avidity and FDG-PET uptake.

(c) The level of TSH suppression should be $<0.1 \mathrm{mU} / \mathrm{l}$.

\section{Narrative of the recommendation}

Persistent Biochemical disease (for definition, see chapter 13). Persistent biochemical disease is observed in about $10-20 \%$ of DTC and in all risk categories. According to $\mathrm{Tg}$ values, this condition is defined as "incomplete biochemical response" (Tg on LT4 $>1 \mathrm{ng} / \mathrm{mL}$ and TSH-stimulated $>10 \mathrm{ng} / \mathrm{mL}$ ) and is different from an "indeterminate response" (with Tg on LT4 $<1 \mathrm{ng} / \mathrm{mL}$ and TSH-stimulated $<10 \mathrm{ng} / \mathrm{mL}$ ) frequently characterized by concomitant non-specific imaging findings. In some patients, persistently high or rising $\mathrm{TgAb}$ levels, in the absence of detectable $\mathrm{Tg}$, indicate persistent disease. Outcome: during follow-up, about $20 \%$ of patients with persistent biochemical disease will progress with appearance of structural disease [51, 52]. Remaining patients will not have evidence of progression during the long-term follow-up, often without any additional treatment. Death rate is $<1 \%$. 
Persistent Structural Disease (see chapter 13): Persistent structural disease is observed in all DTC risk categories with highest prevalence in high-risk tumors. The most common sites of loco-regional disease are cervical lymph nodes. Distant metastases are more frequent in lung followed by bone.

131I treatment is useful in case of iodine avidity. In patients with persistent disease after a cumulative activity of more than $600 \mathrm{mCi}$, an alternative therapy should be considered [53] if the disease is progressing. RAI refractory tumor has a high probability of progression and will require systemic therapy with TKI during follow-up. FDG-PET is useful for prognostic purpose and when tumor lesions are FDG-PET positive prognosis if unfavorable [54].

Whenever systemic treatment is advocated, the decision should not be taken according to strict RECIST criteria. These should be limited to experimental clinical trials.

Tumors with low tumor burden, and also small metastatic lymph nodes should be followed without additional therapy. Localized or single metastases may require an individualized treatment, with surgery or external beam radiotherapy or other therapeutic options as embolization or radiofrequency. About $50-80 \%$ of these patients will have persistent disease during the long-term follow-up despite medical treatment with a specific mortality of $11 \%$ in patients with loco-regional disease and in almost $60 \%$ in patients with distant metastases.

\section{Item 16: Follow-up of patients treated with lobo-isthmectomy}

\section{Recommendation statement}

(a) Following lobo-isthmectomy, cervical US to evaluate the thyroid bed and central and lateral cervical nodal compartments should be performed at 3-6 months and then periodically, according to the patient's risk for recurrent disease and $\mathrm{Tg}$ levels which should be evaluated on thyroid hormone therapy.

(b) In patients treated with lobo-isthmectomy, response to treatment should be evaluated, as follows:

Excellent response: stable non-stimulated $\mathrm{Tg}$ levels, consistent with the presence of a normal thyroid lobe, negative US.

Biochemical incomplete response: elevated non-stimulated $\mathrm{Tg}$ levels not consistent with the presence of a normal thyroid lobe or serum $\mathrm{Tg}$ levels increasing over time in the presence of similar TSH levels, negative US.

Structural incomplete response: structural evidence of disease, regardless of serum $\mathrm{Tg}$.
Indeterminate response: non-specific findings on US and/or Tg trend not assessable.

(c) Diagnostic and therapeutic actions should be based on the response to initial therapy, as follows:

Excellent response: decrease in the intensity and frequency of follow-up;

Biochemical incomplete response: additional imaging and potentially additional therapies;

Structural incomplete response: completion thyroidectomy or ongoing observation according to multiple clinico-pathologic factors including age, size, location, rate of growth, and 18FDG avidity;

Indeterminate response: continued observation with appropriate serial imaging of the non-specific lesions and serum $\mathrm{Tg}$ monitoring.

\section{Narrative of the recommendation}

These recommendations apply to patients submitted to loboisthmectomy. Since the majority of recurrences will occur either in the contralateral lobe or in cervical lymph node metastases, neck ultrasonography remains the key modality to identify disease recurrence. The follow-up with radioactive iodine scanning is not helpful and serum thyroglobulin values are thought to have less value than in patients treated with total thyroidectomy and RAI ablation.

In 2013, Vaisman et al. [55] reported a structural recurrence rate of $7.1 \%$ in a cohort of 70 low-intermediate-risk patients followed for a median of 11 years with thyroglobulin values and serial neck ultrasound examinations after thyroid lobectomy for papillary thyroid cancer. Importantly, $99 \%$ of the patients were subsequently rendered free of disease after identification and treatment (by surgery and/or RAI) of their recurrent disease. The primary modality for detection of structural disease recurrence was neck ultrasound, while a trend in serum $\mathrm{Tg}$ over time that was stable or declining made it very unlikely that disease recurrence would be identified.

Specific cut-off levels of Tg to distinguish normal residual thyroid tissue from persistent thyroid cancer are unknown. Nevertheless, in 2016, Momesso et al. [56] demonstrated that stable, non-stimulated levels $<30 \mathrm{ng} / \mathrm{mL}$, and undetectable $\mathrm{TgAb}$ with negative imaging are suggestive for an excellent response after initial treatment. On the other hand, regardless of the cutoff, rising non-stimulated $\mathrm{Tg}$ are the strongest predictor of recurrent/persistent structurally evident disease.

The presence/absence and/or the trend of $\mathrm{TgAb}$ levels cannot be considered in the follow-up of patients submitted to lobectomy, due to the presence of the residual lobe. 


\section{Item 17: Is there an indication for rhTSH in the preparation of patients undergoing radioiodine therapy for metastatic disease?}

\section{Recommendation statement}

(a) The use of rhTSH is not routinely recommended for radioiodine therapy of loco-regional and distant metastases from DTC because has not been registered for this use.

(b) Recombinant human TSH-mediated therapy can be used according the the Italian law (648/96).

- Patients whose serum TSH cannot be raised (at least $30 \mu \mathrm{UI} / \mathrm{mL}$ ) because of primary or secondary pituitary disease or functional metastasis.

- Patients with underlying comorbidities making iatrogenic hypothyroidism potentially risky:

- previous cerebral stroke or TIA;

- cardiomyopathy (NYHA grade III or IV);

- severe renal failure (stage 3 or superior);

- serious mental disorders (severe depression, psychosis).

(c) Such patients should be given higher activity that would have been given had they been prepared with hypothyroidism or a dosimetrically determined activity.

\section{Narrative of the recommendation}

Although rhTSH has been used "off-label" safely and sometimes with efficacy in hundreds of patients with metastases from DTC, since the drug has not undergone a prospective Phase 3 study for this indication, it has not been registered for this use. In addition, only limited data have been published on radioiodine kinetics in metastatic tissue under rhTSH stimulation and publications to date comprise only case reports or small non-prospective and non-randomized studies. Currently, there are insufficient outcome data to recommend rhTSH-mediated therapy for all patients with distant metastatic disease being treated with ${ }^{131} \mathrm{I}$.

The few published experience usually showed that rhTSH can stimulate the uptake of radioiodine in metastatic lesions, but suggested that stimulation by rhTSH may be less effective than hormone withdrawal in concentrating radioiodine into metastatic lesions. This can be due to several reasons.

- First, the TSH elevation achieved with rhTSH is sharper, but much shorter lived than that attained by THW. More protracted TSH stimulation (as obtained by THW) might result in enhanced DTC cell expression or trafficking of NIS, which might lead to higher radioiodine uptake.
- Second, normal residual thyroid tissue and metastatic tissue are biologically distinct. Neoplastic tissue has much less ability to concentrate iodine and may require more profound TSH stimulation, obtained more through THW than by means of rhTSH administration. However, no comparative data are available on this specific issue.

- Finally, the markedly faster (by $\sim 50 \%$ ) renal excretion of radioiodine under euthyroid conditions (rhTSH administration) compared to hypothyroid conditions (THW) raises the possibility that the radiation absorbed dose (Gy delivered to the lesions) by a given ${ }^{131}$ I activity could differ appreciably between the two TSH stimulation methods. Freudenberg et al. [57] reported a delivered lower radiation doses per GBq of administered activity after rhTSH than after THW in distant metastases. Similar results have been reported in a small pilot study by Pötzi et al. [58] using lesion cumulative activity in $\mu \mathrm{Ci}$ as a surrogate marker for lesion dose. Though visual analysis of the rhTSH post-therapy scans showed concentration of ${ }^{131}$ I in most metastatic sites, Rani et al. [59] recently reported that the mean effective half-life of 131I, the mean 24-h \% uptake and the mean tumor radiation absorbed dose per administered $\mathrm{MBq}(\mathrm{mCi})$ in lung or skeletal metastases was less during the rhTSH protocol than THW protocol. Despite these evidences, the results obtained by Tala et al. [60] in two groups of metastatic patients treated with 131-I and prepared with rhTSH or TWH were similar, but the study has several limitations including a four injection regimen of rhTSH administration.

These controversial and inaccurate data are the main reasons for which rhTSH cannot be recommended yet for the preparation of radioiodine treatment in patients with known, or at high risk of having, neoplastic foci, except those who cannot tolerate withdrawal for underlying comorbidities (e.g., previous cerebral stroke or TIA, cardiomyopathy, strict renal failure or serious mental disorders) or patients whose serum TSH cannot be raised because of primary or secondary pituitary disease or functioning metastases. In Italy, the Italian Medicines Agency (AIFA) approved this use in patients with metastatic disease, defining the new indications and the additional criteria for reimbursement of rhTSH on the basis of law 648/96.

Because significant morbidity from swelling of metastatic tissue following rhTSH administration has been described, rhTSH needs to be used cautiously in patients with metastases in "closed spaces" in the body, such as brain or spine. The institution of temporary high-dose corticosteroid therapy is recommended for trying to limit the risk of acute tumor swelling, probably depending on a higher vascularization of the lesion with increasing of the edema than a real tumor mass growing. 
A large, well-controlled, prospective, within patient study is needed to resolve the clinically important item of whether lesion doses significantly differ between the two TSH stimulation methods. Until data from such a study become available, we suggest reserving rhTSH-aided treatment of metastatic DTC to patients who are unable to tolerate THW or to increase TSH levels by TSH endogenous stimulation.

\section{Item 18: Radioiodine treatment of metastatic disease: standard doses vs. dosimetry, maximal cumulative activity, frequency of treatment courses}

\section{Recommendation statement}

(a) The selection of radioiodine activity to administer in the treatment of loco-regional or distant metastases from DTC can be determined empirically (fixed or empiric activities ranging from 3.7 to $7.4 \mathrm{GBq}, 100$ $200 \mathrm{mCi}$ ) or by dosimetry.

(b) Empiric activities ranging between 3.7 and $7.4 \mathrm{GBq}$ (100-200 mCi) appears to be a reasonable option for most patients. Amounts of ${ }^{131} \mathrm{I}$ exceeding $7.4 \mathrm{GBq}$ $(200 \mathrm{mCi})$ are not recommended in elderly patients ( $>70$ years) and patients with renal insufficiency because the maximum tolerated radiation absorbed dose ( 2 Gy to the blood) is potentially exceeded.

In these patients, dosimetry could be considered.

(c) The total number of treatments that can be performed in the same patient and the time occurring between two treatments cannot be established in a strict way. The decision must be individualized and based on several factors, including the disease response to treatment (e.g., decreasing of the size/number of the lesions and/ or serum $\mathrm{Tg}$ levels), the age of the patient and the onset of deterministic effects (e.g., radiation lung damage, bone-marrow suppression and salivary gland damage). However, retreatment should be preferably performed not earlier than 6-12 months from previous treatment.

\section{Narrative of the recommendation}

Although there are theoretical advantages to prefer dosimetry, to date, outcome data are insufficient and no recommendation can be made about the superiority of dosimetry over fixed activities. Dosimetry plays an important role in selected cases (e.g., patients with or extensive pulmonary metastases, elderly patients or patients with unusual situations such as renal insufficiency) and it is exceptionally helpful in clinical trials.

Three basic approaches have been described to select the activity of radioiodine for treatment of metastatic disease.

\section{Fixed or empiric activities}

All patients with a given clinical situation (e.g., lymph node, lung or bone metastases) get the same radioiodine activity [e.g., $3.7 \mathrm{GBq}(100 \mathrm{mCi})-7.4 \mathrm{GBq}(200 \mathrm{mCi})]$. This is the easiest and common approach used in clinical practice, with a long history of use, a high grade of convenience and a reasonably acceptable rate and severity of complications. However, given the heterogeneity of patients with metastatic disease, this is clearly the most arbitrary method of selecting therapy that has the potential to under treat some patient while over treating others.

\section{Bone-marrow (blood) dosimetry}

The second approach is to use quantitative methods to determine the maximum tolerated activity (MTA) of radioiodine that could be administered within a safe upper limit of absorbed dose to non-target tissues, such as the bone marrow or lungs in the presence of diffuse metastases. With this technique, the ${ }^{131}$ I activity is calculated to deliver a maximum of 2 Gy to the whole blood to avoid serious myelosuppression. In addition, the whole-body retention at $48 \mathrm{~h}$ after radioiodine administration should not exceed $4.4 \mathrm{GBq}$ $(120 \mathrm{mCi})$ in the absence of iodine-avid diffuse lung metastases or $2.96 \mathrm{GBq}(80 \mathrm{mCi})$ in the presence of such lesions. The theoretical rationale for administration of the MTA is based on the concept that a maximal safe prescribed activity should have greater therapeutic benefit than multiple smaller empiric activities that may induce nonlethal changes in the cancer tissue with subsequent cellular repair. However, the approach does not estimate the absorbed radiation dose to the metastasis and the MTA may be given without any therapeutic effect.

\section{Lesion-based dosimetry}

The objective of this method is to determine the radioiodine activity that delivers the recommended absorbed radiation dose to the metastases whilst minimizing the risk to the patient. The traditionally recommended radiation absorbed dose to successfully treat metastatic disease is $80-120$ Gy ( $80 \%$ control rate) [61]. The strength of this approach is a more selective exposure to individual patients based upon their individual needs without an increase in radiation exposure to the patient population. However, several disadvantages of the quantitative tumor dosimetry are reported including the incomplete tumor destruction due to the wide range of absorbed lesion doses within a single patient and within different lesions, the impossibility to accurately estimate the lesion mass in case of disseminated metastases or irregularly shaped lesions, the systematic absorbed doses 
underestimation for lesions $<5 \mathrm{~mm}$ in diameter and/or with low radioiodine uptake.

In the only retrospective comparison between fixed activity and dosimetry, no difference in the patient's outcome has been found [62]. In general, patient-specific dosimetry requires a number of scintigraphic scans and staff resources. Several factors can limit the implementation of patient-specific dosimetry, such as shortage of knowledge and knowhow, shortage of medical physicists working in nuclear medicine, limited access to scanner and dedicated software.

In case that dosimetry is preferred, the administered activity calculated on the lesions should be lowered if the MTA to the blood is inferior to that activity.

\section{Item 19: "Empiric" treatment with radioiodine in $\mathrm{Tg}$ positive/diagnostic WBS negative patients: still an indication?}

\section{Recommendation statement}

(a) Empiric treatment with RAI $(100-150 \mathrm{mCi}, 3.7-$ $5.2 \mathrm{mBq}$ ) may be considered in patients with elevated serum $\mathrm{Tg}$ levels or rapidly rising serum $\mathrm{Tg}$ levels or rising anti-Tg antibody levels and negative macroscopic structural (US, TC, RMI) or metabolic (FDG-PET/CT) imaging.

(b) If the post-therapy scan is negative and structural disease is evident, further RAI therapy should never be used and the patient should be classified as RAI refractory.

(c) On the contrary, if the post-therapy scan shows the presence of metastatic disease significantly taking up radioiodine, further treatment courses can be considered as long as evidence of clinical benefit is provided.

\section{Narrative of the recommendations}

After a period of great enthusiasm in favor of empiric treatment with RAI in Tg positive/scan negative patients [63], recent data have indicated that the benefit from such therapy are almost negligible [64]. Thus, the indication for empiric RAI therapy has been dramatically limited to very selected patients. In addition, one has to keep in mind that this empiric approach does not fulfill the first principle of radiological exposure according to which any radiological procedure or therapy must be "justified". Furthermore, no target dosimetry is possible under these circumstances. ${ }^{124} \mathrm{I}$ PET/TC could offer more information in these patients.

The objective of empiric RAI therapy is mainly to aid in disease localization, and, hopefully shrinkage of the tumor. The first aim is achieved in one-third of patients. Therapeutic benefits are reported in a minority of patients either as a decline in serum Tg or stabilization of tumor size, particularly in case of micro-metastases in the lung. However, there is no evidence for improved survival.

Two main factors should be considered for decision making: the level of serum Tg elevation and the results of ${ }^{18}$ FDG-PET. As far as the Tg levels are concerned, there is no consensus of the serum Tg cutoff above which an empiric dose of RAI should be delivered. In addition, evidence is provided that slightly elevated stimulated serum $\mathrm{Tg}(<10 \mathrm{ng} /$ $\mathrm{mL}$ ) may decrease spontaneously, without any therapy, during follow-up. A good compromise is to use cut-off levels of serum $\mathrm{Tg}$ above which a spontaneous decline is unlikely to occur, particularly when these serum $\mathrm{Tg}$ levels or $\mathrm{TgAb}$ levels are rising over time.

Metastatic lesions that are ${ }^{18}$ FDG-PET positive generally do not concentrate RAI. In this setting, RAI therapy is unlikely to be beneficial and because of this, it is recommended that empiric RAI therapy should be administered mainly in patients with negative ${ }^{18}$ FDG-PET.

\section{Item 20: Adverse events of radioiodine therapy}

\section{Recommendation statement}

(a) Nausea, taste disturbance and salivary gland swelling are the most common early side effects. Recommended measures to prevent damage to the salivary glands include hydration and sour candies or lemon juice administration.

(b) Women of childbearing age should avoid pregnancy for 6-12 months after receiving radioiodine.

(c) Depending on the clinical situation, radioiodine therapy could be deferred until lactating women have stopped breastfeeding or pumping for at least 3 months.

(d) Men receiving cumulative radioiodine activities $\geq 400 \mathrm{mCi}$ should be counseled on potential risks of infertility.

(e) Although present, the risk of developing a second primary malignancy (SPMs) due to radioiodine therapy in long-term survivors is considered very small and related to the cumulative activity. There is no evidence of increased risk of SPMs after a single administration of radioiodine for ablative treatment.

\section{Narrative of the recommendation}

Radioiodine therapy is generally well-tolerated, and in most cases, the early deterministic effects are of scarce clinical relevance. However, both the choice of the single and cumulative activities and the patient's preparation (e.g., using laxatives, oral hydration, diuretics or a short course of steroids) 
should be accurate to minimize the risk of early and late side effects.

The main deterministic effects of radioiodine therapy in patients with DTC can concern the salivary glands (taste disturbance, swelling, sialoadenitis, xerostomia, salivary duct obstruction), eye and nasolacrimal gland (xerophthalmia, epiphora, conjunctivitis), genital system (hypospermia and azoospermia in males and temporary amenorrhea or oligomenorrhea in females), lungs (very rare acute radiation pneumonitis and fibrosis) and bone marrow (neutropenia, low platelet count and anemia) [65]. Most of these effects are early and transient, but some of them may become permanent and appear only months or years after treatment, such as bone-marrow depression, radiation pulmonary fibrosis, early onset of menopausa in women, oligospermia in men and chronic dry eye.

Objective salivary gland dysfunction [66] (abnormal scintigraphic findings, defined as decreased salivary secretion and excretion fraction) are significantly more frequent observed after I-131 therapy when compared intra-individually (before I-131) and when compared to controls without exposure to I-131. The prevalence of objective salivary dysfunction after I-131 ranged from 37 to $72 \%$. The administered cumulative I-131 activity in relation to objective salivary gland dysfunction is an important risk factor. The prevalence of symptomatic salivary gland dysfunction is lower than objective salivary gland dysfunction (16-54 vs. $37-72 \%$, respectively) indicating that objective salivary dysfunction may be present in the absence of symptoms. Symptomatic salivary gland dysfunction correlated well with the cumulative I-131 activity.

Objective lacrimal gland dysfunction is defined as an abnormal functional tear test (Schirmer's tear test, tear film break-up time, etc.). An abnormal Schirmer's tear test is found significantly more frequently in patients exposed to I-131 compared to unexposed patients.

Regarding male gonadal dysfunction [67] the prevalence of oligospermia after exposure to I-131 ranges from 25 to $50 \%$. Follicle-stimulating hormone (FSH) concentrations are significantly increased (from 35 to $100 \%$ in respect to normal range) after I-131 therapy compared to the values measured before exposure to I-131, with a maximal FSH increase 6 months after I-131 therapy. On average, mean concentrations of serum FSH has normalized 18 months after the last I-131 treatment. However, for patients treated with high activities of I-131, the risk to develop persistent elevated serum FSH concentrations is increased. Mean serum luteinizing hormone $(\mathrm{LH})$ concentrations are transiently increased after I-131 therapy. Serum testosterone concentrations are not significantly changed.

Female gonadal function evaluation reveals temporary amenorrhea and menstrual irregularities in $20-27 \%$ of patients [68]. In one study, a significant younger menopausal age in DTC patients who received I-131 compared to subjects who did not receive I-131 therapy is observed (49.5 vs. 51.0 years). Most studies have demonstrated that the birthrate in female patients is not affected by the treatment with I-131. The reduced birthrate reported by other studies may be explained by a possible impact of I-131 therapy on both reproductive choice and health. In one study, it has been observed that the overall median time to first delivery following initial presentation of DTC is significantly longer in women who received I-131 therapy compared to patients who did not (34.5 vs. 26.1 months). In addition in this case, it could not be excluded that this delay may have been influenced by physicians' recommendations to avoid pregnancy for 6-12 months after I-131 therapy or may have been related to hormonal imbalances caused by hypothyroid state. I-131 therapy is not a risk factor to bear a miscarriage or preterm birth deliveries in pregnancies occurring after I-131 administration. In one study, however, significantly more preterm deliveries were found in patients who received I-131 therapy compared to patients who did not (9.1 vs. 1.4\%). No increased incidence of congenital abnormalities in the offspring of women previously treated with $\mathrm{I}-131$ is found.

The possible induction of second primary malignancies (SPMs) is one of the most relevant untoward effects of radioiodine treatment particularly when the cumulative activity was $>37 \mathrm{GBq}(>100 \mathrm{mCi}$ ) [69]. A meta-analysis of two large multicenter studies showed that the relative risk (RR) of SPMs after radioiodine therapy (relative to thyroid cancer survivors not treated with radioiodine) was significantly increased for solid tumors and leukemia at 1.19 ([95\% CI 1.04-1.36], $p<0.010$ ) and 2.5 ([95\% CI 1.13-5.53], $p<0.024)$, respectively. However, in all studies the absolute increase in second primary malignancy risk attributable to radioiodine is considered to be very small. These studies showed an increased risk linked to increasing doses of radioiodine for salivary glands, digestive tract and soft-tissue and bone sarcomas. A study on patients with low-risk DTC (T1N0 PTC) from the SEER registry concluded that the excess risk of leukemia after radioiodine therapy was significantly greater (standardized incidence rate of 5.32 [95\% CI 2.75-9.30] in young individuals (aged $<45$ ) compared with older individuals (standardized incidence rate of 2.26 [95\% CI 1.43-3.39]

\section{Item 21: Definition of RAI refractory disease}

\section{Recommendation statement}

(a) A thyroid cancer can be defined as RAI refractory when at least one of the following patterns is verified: 
- Evidence of measureable metastatic lesion(s) not able to take up radioiodine, as demonstrated by a negative post-therapeutic 131-I whole-body scan.

- Evidence of measureable metastatic lesion(s) not able to take up radioiodine, as demonstrated by a negative 131-I whole-body scan, even if accompanied by other lesions still able to take up iodine.

- Evidence of measureable metastatic lesion(s) that, despite their ability to take up iodine, are growing between one 131-I treatment and the following, thus indicating the ineffectiveness of 131-I therapy.

\section{Narrative of the recommendation}

Differentiated thyroid cancer (DTC) is considered as a tumor with an excellent prognosis, with a 93-95\% survival rate. The initial therapy, in particular for intermediate and highrisk patients, is total thyroidectomy (TTx) plus/minus radioiodine remnant ablation (RRA). In the majority of cases, this initial therapy is sufficient to cure the patients. About $5 \%$ of patients with DTC show distant metastases at the time of the diagnosis. 131-I therapy is the therapy of choice and treatments should be repeated until the evidence of 131-I uptake. About $30 \%$ of these patients will be cured with this therapy while the other sooner or later will become RAI refractory and will require other therapies. The overall survival of this group of patients may be significantly lower when compared with other groups that retain the ability to take up radioiodine.

There are evidences that the mean cumulative activity of 131-I within which we can expect to cure the metastatic DTC patient is $22 \mathrm{GBq}(600 \mathrm{mCi})$. For this reason, several authors suggest that after this total activity is reached the 131-I treatments must be stopped and the tumor can be considered RAI resistant or refractory. Although everybody agrees that patients who are not cured before reaching this cumulative activity of 131-I will likely never cured by 131-I, several experts are convinced that those patients who showed a "stable disease" (i.e., same size and same number of lesions) under 131-I repeated treatments (every 12-18 months) should not be considered as RAI resistant or refractory. These patients should be continued to be treated with 131-I until the evidence of clinical benefit, and the stability of the disease can be considered a clinical benefit, particularly if the patient is in good general health conditions.

In patients with metastatic DTC, it is not rare to observe the heterogeneous behavior of RAI uptake in different metastatic lesions. This pattern is rather typical in patients with large and multiple metastases as demonstrated in studies with ${ }^{124 I}$-PET-CT scan [70]. In these cases is rather frequent to observe the "flip-flop" phenomenon that describes the situation of simultaneous metastatic lesions that are either 18FDG-PET negative but 131-I WBS positive or
18FDGPET positive but 131-I WBS negative. Moreover, it has been well demonstrated that the 18FDG PET positivity is "per se" a poor prognostic factor for survival independent from the ability of the metastatic lesions to take up iodine [54]. On the basis of these observations, 18 FDG-PET positive metastatic lesions can be considered as RAI resistant or refractory.

\section{Item 22: What is the role of systemic therapy (kinase inhibitors, conventional chemotherapy) in treating metastatic DTC?"}

\section{Recommendation statement}

(a) Before selecting systemic therapies, a rigorous screening should be performed to assess whether one or more lesions are candidate to local therapies.

(b) Patients with locally advanced and/or metastatic RAI refractory disease, rapidly progressive, significantly symptomatic and/or with life threatening lesions not suitable for local therapies should be considered for systemic treatment.

(c) The major roles of systemic therapy is to reduce the growth rate and/or to reduce the tumor burden to improve the progression free survival of patients and, possibly, to control the symptoms related to the neoplastic disease.

(d) Nowadays, multikinase inhibitors approved by FDA and EMA (i.e., sorafenib and lenvatinib) are considered as the first line systemic therapy for advanced, RAI refractory and progressive DTC.

(e) Traditional chemotherapy is no more indicated with the exception of cases in whom the TKI are ineffective or cannot be used.

(f) Patients with stable RAI refractory metastatic disease without any symptoms, with a slow progression during the follow-up and without metastatic lesions at risk of life may be monitored with active surveillance at regular intervals (3-12 months) by cross-sectional imaging under TSH suppressive thyroid hormone therapy.

\section{Narrative of the recommendation}

Systemic therapy should be initiated when the progression of the local or metastatic disease is documented with radiological imaging and according to Response Evaluation Criteria in Solid Tumors (RECIST). In some cases (i.e., large tumor burden, high risk of compression or infiltration of neighboring organs), the systemic therapy can be initiated even in the absence of a proved progression.

However, whenever the progression of the disease or its riskiness is related to a single lesion treatable with a local 
treatment selective therapies (i.e., thermoablation, external radiotherapy, ethanol injection, chemoembolization, local surgical treatment etc.) are better indicated and strongly suggested

At present, tyrosine kinase inhibitors (TKI) represent the first choice systemic therapy since chemotherapy, both as single agent or in combination, has never given a durable clinical benefit. Two drugs are nowadays available, sorafenib [71] and lenvatinib [72], which have been demonstrated to significantly improve the progression free survival of patients treated with the drug with respect to those treated with placebo. Moreover, both studies showed also a significant increase of the objective response rate in patients treated with the drugs with respect to patients treated with placebo. However, it should be noted that a prolongation of survival has never been demonstrated in clinical trials. This does not mean that there is no survival benefit, due to the bias of the possibility for the patients in the placebo group to shift to TKI when the blind has been open.in clinical trials

When disease progression is demonstrated during TKI therapy, the shift to another TKI is indicated based on evidence that the second drug has high chance of being effective.

Since TKI are cytostatic but not cytotoxic drugs, they should be started when the disease is progressing. However, since they are also potent antiangiogenic drugs, a rapid devascularization and consequent shrinkage of tumor lesions is expected. This latter activity can have also a significant impact on symptoms due to the pressure or infiltration of other organs.

\section{Item 23: L-Thyroxine therapy after total thyroidectomy or lobo-isthmectomy}

\section{Recommendation statement}

\section{Degree of TSH suppression after initial treatment}

The degree of initial TSH suppression depends on the risk classification after initial treatment. Age and comorbidities should also be considered.

(a) ATA high-risk thyroid cancer patients: initial TSH suppression to $\leq 0.1 \mathrm{mU} / \mathrm{L}$, unless contraindicated by concurrent diseases;

(b) ATA intermediate-risk thyroid cancer patients: initial TSH suppression to $0.1-0.5 \mathrm{mU} / \mathrm{L}$;

(c) ATA low-risk patients who have undergone or not remnant ablation and have undetectable serum Tg levels: TSH in the normal range $(0.5-2 \mathrm{mU} / \mathrm{L})$;

(d) ATA low-risk patients who have undergone remnant ablation and have low serum Tg levels: TSH may be maintained at or slightly below the lower limit of normal (0.1-0.5 mU/L);

(e) ATA low-risk patients who have undergone lobectomy: $\mathrm{TSH}$ may be maintained in the mid to lower reference range $(0.5-2 \mathrm{mU} / \mathrm{L})$.

Note: In patients treated by lobectomy and living in an iodine deficient country, L-thyroxine treatment may be considered to reduce the TSH-induced thyroid nodular hyperplasia of the residual lobe, considering, age, TSH levels and ultrasound feature of the remaining lobe.

\section{Degree of TSH suppression during follow-up [1]}

The degree of TSH suppression depends on the ongoing risk classification. Age and comorbidities should also be considered.

(a) Patients with a structural incomplete response to therapy, regardless of the initial ATA risk classification: $\mathrm{TSH}$ should be maintained $\leq 0.1 \mathrm{mU} / \mathrm{L}$, in the absence of specific contraindications.

(b) Patients with a biochemical incomplete or indeterminate response to therapy, regardless of the initial ATA risk classification: TSH should be maintained between 0.1 and $0.5 \mathrm{mU} / \mathrm{L}$, in the absence of specific contraindications.

(c) Patients with an excellent (clinically and biochemically free of disease) response to treatment, regardless of the initial ATA risk classification: TSH may be kept within the low-normal reference range ( 0.5 and $2 \mathrm{mU} / \mathrm{L})$.

(d) Patients who have not undergone remnant ablation or adjuvant therapy with a normal neck US, and low or undetectable serum $\mathrm{Tg}$, and $\mathrm{Tg}$ or $\mathrm{TgAb}$ that are not rising: TSH can be allowed to rise to the reference range (0.5 and $2 \mathrm{mU} / \mathrm{L})$ (Fig. 1).

\section{Narrative of the recommendation}

Retrospective and prospective studies have demonstrated that TSH suppression to below $0.1 \mathrm{mU} / \mathrm{L}$ may improve outcomes in high-risk thyroid cancer patients, though no such evidence of benefit has been documented in low-risk patients. Higher degrees of suppression to $<0.03 \mathrm{mU} / \mathrm{L}$ may offer no additional benefit. Almost all the available data on the use of thyroid hormone suppression therapy refer to patients submitted to total thyroidectomy, whereas there is little or none available data to guide TSH targets in low-risk patients who have undergone lobectomy.

Adverse effects of TSH suppression may include the known consequences of subclinical thyrotoxicosis, including exacerbation of angina in patients with ischemic heart disease, increased risk for atrial fibrillation in older patients, 

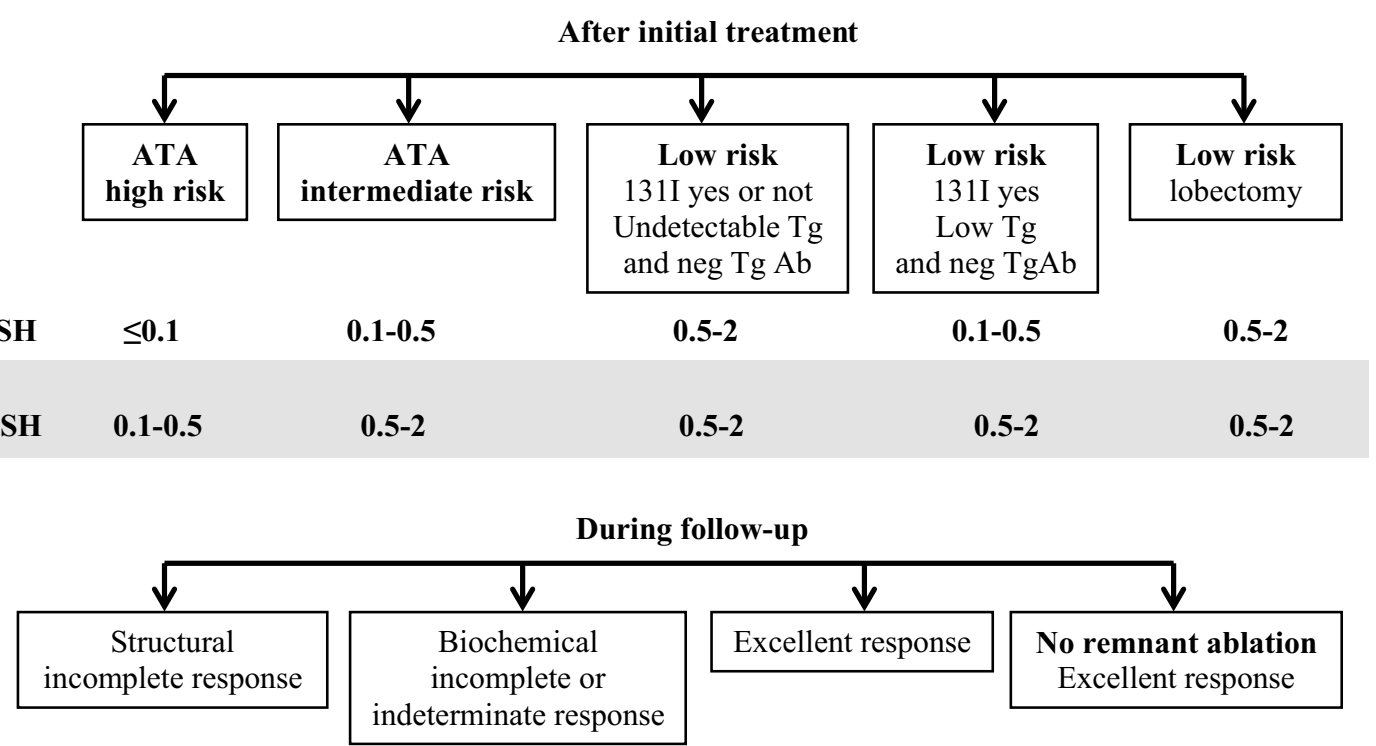

$\begin{array}{lllll}\text { TSH } & \leq 0.1 & 0.1-0.5 & 0.5-2 & 0.5-2\end{array}$

Comorbidities

and/or age $>65$ yrs

$\begin{array}{lll}\text { TSH } & 0.1-0.5 & 0.5-2\end{array}$

$0.5-2$

$0.5-2$

Fig. 1 Goal TSH levels after initial treatment and during follow-up according to the risk stratification

and increased risk of osteoporosis in postmenopausal women. Thus, a balance is needed between the potential benefit of TSH suppression on tumor recurrence or progression and the risks of subclinical thyrotoxicosis, especially in patients at high risk of adverse effects on heart and bone. When a TSH suppressive treatment has an absolute indication, adjunctive therapy with calcium supplements, vitamin $\mathrm{D}$, and other bone enhancing agents (bisphosphonates, denosumab, etc.) should be considered in peri- and postmenopausal women at risk for bone loss.

Finally, the appropriate degree of TSH suppression by thyroid hormone therapy during follow-up is still unknown, especially in high-risk patients who become free of disease after initial treatment. This is because discordant data are available, some studies indicating that the degree of TSH suppression is an independent predictor of recurrence, and others showing that the degree of TSH suppression does not correlated with outcome.

\section{Item 24: Legal issue in the management of thyroid cancer}

\section{Background}

The frequency of medical-legal issues in the management of thyroid cancer is rising everywhere in the world and also in Italy, particularly in the setting of surgical treatment.
Knowledge of the character and frequency of such problem following surgery of the thyroid gland is scant; however, it is apparent that post-operative recurrent laryngeal nerve palsy and hypoparathyroidism are the principal causes of surgical malpractice claims. There are no specific data regarding the extent of the medical-legal controversies regarding thyroid cancer management in Italy.

\section{Recommendation statement}

(a) National clinical practice guidelines may not necessarily constitute a legal standard of care in jurisdiction.

(b) In Italy, a law is currently under discussion regarding regulation concerning professional responsibility of health care personnel. This law provides a number of changes from the law currently in force, especially with regard to the criminalization of medical procedures. It establishes a set of rules that would regulate conflicts in the forensic field.

(c) A good informed consent is the basis for lowering medical conflict. It is important to establish a relationship of alliance and empathy between doctor and patient.

\section{Narrative of the recommendation}

This document is intended to inform clinical decision making. A major goal of this consensus statement is to minimize potential harm from overtreatment in a majority of patients 
at low risk for disease-specific mortality and morbidity, while appropriately treating and monitoring those patients at higher risk. This document should not be interpreted as a replacement for clinical judgement and should be used to complement informed, shared patient-health care provider deliberation on complex issues.

The safety of treatment is an integral part of the right to health care and is pursued in the interest of the individual and the community.

(Criminal liability of the operator in the health care profession). The operator of the health care profession that, in carrying out his or her activities, due to inexperience, causes death or personal injury of the patient is liable for offenses referred to only in cases of gross negligence. The effects of the provisions of the first paragraph exclude gross negligence when, subject to the relevant specificities of the case, they comply with good clinical care practices and recommendations provided by the guidelines, as defined and published in accordance with the law.

Informed consent: patients should be adequately informed by the operator of the indications for an individual treatment or possible alternative treatments, the expected advantages from the therapy, its general and specific complications and possible rehabilitation therapy, as well as the clinical consequences of potential permanent injuries, particularly after surgery. The information provided should be clearly explained, complete and prompt. After providing the most complete information, the physician will seek the patient's consent to perform therapy, especially taking into full consideration any expression of dissent, even on individual aspects of the procedure or its potential consequences [73, 74]. Transmission of information and the informed consent should preliminarily take place at the initial outpatient visit and be renewed at admission, especially if enough time has passed such that the initial conditions may have changed. In fact, the patient must be given the opportunity to discuss in depth with his/her physician (or other trusted persons) the information received and, if desired, get information on the health facility where he or she will be treated and/or on the team that will perform therapy. Given the peculiarity of the therapeutic intervention in case of surgery (partial or total removal of thyroid glands and neck nodes, central o lateral) and its potential consequences on the physical integrity of the subject, it is necessary that written documentation of the informed and voluntary consent be retained, and that the informed consent process be documented in a specific chart note.

\section{Compliance with ethical standards}

Conflict of interest The authors have no conflict of interest to disclose.
Ethical approval This study complies with ethical standard of research. It does not involve data or treatment of human being nor animals.

Informed consent For this type of study informed consent is not required.

\section{Appendix 1: Reporting form for thyroid tumors}

\section{Gross description (macroscopic)}

Thyroid

Weight (size)

Multinodularity/uninodularity

Macroscopic appearance of the nodule:

- diameter

- homogeneous/heterogeneous

- tumor color

- expansive margins/infiltrative margins

- presence/absence of a capsule

- solid/colloid

- cystic-hemorrhagic regression

- calcifications (pattern and location).

Site

- right lobe

- left lobe

- isthmus

- pyramidal lobe

Distance from thyroid capsule/surgical margins

\section{Lymph nodes}

Site

Size of the specimen

Gross features of the largest nodes.

\section{Microscopic examination}

\section{Thyroid nodule}

Capsule (present/absent)

Structure (colloid/sclerotic/solid)

Histotype

NIFT-P

Papillary 
Classical variant

Follicular variant

Follicular

Minimally invasive

Widely invasive

Oncocytic

Poorly differentiated (insular)

Anaplastic

Medullary

Thyroid lymphoma

Secondary tumors

Aggressive variants of PTC:

- hobnail (report the presence)

- tall cells (more than 10\%)

- solid (percentage value)

- columnar cell (more than 10\%)

- diffuse sclerosing (report the presence)

Capsular invasion (tumor capsule/thyroid capsule)

Vascular invasion (no. of vessels: 4 or more) [19]

Minimal muscular invasion (strap muscles)

Extrathyroidal invasion (subcutaneous soft tissues, larynx, trachea, esophagus, recurrent laryngeal nerve, prevertebral fascia, neck muscles, large vessels) [20]

Immunohistochemistry and molecular analysis (if performed).

\section{Lymph nodes}

Total number

Number of metastatic nodes

Size of the largest metastatic area within the node, micro$(\leq 2 \mathrm{~mm})$ or macrometastases Presence of extranodal invasion [75-77].

\section{References}

1. Haugen BR, Alexander EK, Bible KC et al (2015) American thyroid association management guidelines for adult patients with thyroid nodules and differentiated thyroid cancer the american thyroid association guidelines task force on thyroid nodules and differentiated thyroid cancer. Thyroid. https://doi.org/10.1089/ thy. 2015.0020

2. Leenhardt L, Erdogan MF, Hegedus L, Mandel SJ, Paschke R, Rago T et al (2013) 2013 European thyroid association guidelines for cervical ultrasound scan and ultrasound-guided techniques in the postoperative management of patients with thyroid cancer. Eur Thyroid J. 2(3):147-159

3. Nardi F, Basolo F, Crescenzi A, Fadda G, Frasoldati A, Orlandi F, Palombini L, Papini E, Zini M, Pontecorvi A, Vitti P (2014)
Italian consensus for the classification and reporting of thyroid cytology. J Endocrinol Investig 37(6):593-599. https://doi. org/10.1007/s40618-014-0062-0

4. Ali SZ, Cibas ES (eds) (2010) The Bethesda system for reporting thyroid cytopathology: definitions, criteria and explanatory notes. Springer, New York

5. Perros P, Boelaert K, Colley S, Evans C, Evans RM, Gerrard Ba G, Gilbert J, Harrison B, Johnson SJ, Giles TE, Moss L, Lewington V, Newbold K, Taylor J, Thakker RV, Watkinson J, Williams GR, British Thyroid Association (2014) Guidelines for the management of thyroid cancer. Clin Endocrinol (Oxf) 81(Suppl 1):1-122

6. Yeh MW, Bauer AJ, Bernet VA et al (2015) American thyroid association statement on preoperative imaging for thyroid cancer surgery. Thyroid 25:3-13

7. Wu LM, Gu HY, Qu XH, Zheng J, Zhang W, Yin Y, Xu JR (2012) The accuracy of ultrasonography in the preoperative diagnosis of cervical lymph node metastasis in patients with papillary thyroid carcinoma: a meta-analysis. Eur J Radiol 81(8):1798-1805

8. Tufano RP, Clayman G, Heller KS, Inabnet WB, Kebebew E, Shaha A, Steward DL, Tuttle RM (2015) American thyroid association surgical affairs committee writing task force management of recurrent/persistent nodal disease in patients with differentiated thyroid cancer: a critical review of the risks and benefits of surgical intervention versus active surveillance. Thyroid 25(1):15-27

9. Bilimoria KY, Bentrem DJ, Ko CY, Stewart AK, Winchester DP, Talamonti MS, Sturgeon C (2007) Extent of surgery affects survival for papillary thyroid cancer. Ann Surg 246:375-381

10. Matsuzu K, Sugino K, Masudo K, Nagahama M, Kitagawa W, Shibuya H, Ohkuwa K, Uruno T, Suzuki A, Magoshi S, Akaishi J, Masaki C, Kawano M, Suganuma N, Rino Y, Masuda M, Kameyama K, Takami H, Ito K (2014) Thyroid lobectomy for papillary thyroid cancer: long-term follow-up study of 1,088 cases. World J Surg 38:68-79

11. Adam MA, Pura J, Gu L, Dinan MA, Tyler DS, Reed SD, Scheri R, Roman SA, Sosa JA (2014) Extent of surgery for papillary thyroid cancer is not associated with survival: an analysis of 61,775 patients. Ann Surg 260:601-605

12. Ito $Y$, Miyauchi A, Inoue H, Fukushima M, Kihara M, Higashiyama T, Tomoda C, Takamura Y, Kobayashi K, Miya A (2010) An observational trial for papillary thyroid microcarcinoma in Japanese patients. World J Surg 4(1):28

13. Nikiforov YE, Seethala RR, Tallini G, Baloch ZW, Basolo F, Thompson LD, Barletta JA, Wenig BM, Al Ghuzlan A, Kakudo K, Giordano TJ, Alves VA, Khanafshar E, Asa SL, El-Naggar AK, Gooding WE, Hodak SP, Lloyd RV, Maytal G, Mete O, Nikiforova MN, Nosé V, Papotti M, Poller DN, Sadow PM, Tischler AS, Tuttle RM, Wall KB, LiVolsi VA, Randolph GW, Ghossein RA (2016) Nomenclature revision for encapsulated follicular variant of papillary thyroid carcinoma: a paradigm shift to reduce overtreatment of indolent tumors. JAMA Oncol 2:1023-1029

14. Glockzin G, Hornung M, Kienle K et al (2012) Completion thyroidectomy: effect of timing on clinical complications and oncologic outcome in patients with differentiated thyroid cancer. World J Surg 36:1168-1173

15. Hartl DM, Mamelle E, Borget I, Leboulleux S, Mirghani H, Schlumberger M (2013) Influence of prophylactic neck dissection on rate of retreatment for papillary thyroid carcinoma. World J Surg 37:1951-1958

16. Viola D, Materazzi G, Valerio L, Molinaro E, Agate L, Faviana P, Seccia V, Sensi E, Romei C, Piaggi P, Torregrossa L, SellariFranceschini S, Basolo F, Vitti P, Elisei R, Miccoli P (2015) Prophylactic central compartment lymph node dissection in papillary thyroid carcinoma: clinical implications derived from the first prospective randomized controlled single institution study. J Clin Endocrinol Metab 100:1316-1324 
17. Qu N, Zhang L, Ji QH, Chen JY, Zhu YX, Cao YM, Shen Q (2015) Risk factors for central compartment lymph node metastasis in papillary thyroid microcarcinoma: a meta-analysis. World J Surg 39:2459-2470

18. Raffaelli M, De Crea C, Sessa L, Fadda G, Bellantone C, Lombardi CP (2015) Ipsilateral central neck dissection plus frozen section examination versus prophylactic bilateral central neck dissection in cN0 papillary thyroid carcinoma. Ann Surg Oncol 22:2032-2308

19. Collini P, Sampietro G, Pilotti S (2004) Extensive vascular invasion is a marker of risk of relapse in encapsulated non-Hürthle cell follicular carcinoma of the thyroid gland: a clinicopathological study of 18 consecutive cases from a single institution with a 11-year median follow-up. Histopathology 44:35-39

20. Mahul B, Amin SE, Greene FL, Schilsky RL, Byrd DR, Gaspar LE, Washington MK, Gershenwald JE, Compton CC, Hess KR (2017) In: Brierley JD, Gospodarowicz MK, Wittekind C (eds) 2016 AJCC 8th Edition Cancer Staging Manual. Springer International Publishing AG, Cham, Switzerland.Edition). Wiley Blackwell, Oxford

21. Ganly I, Ibrahimpasic T, Rivera M et al (2012) Prognostic implication of papillary thyroid carcinoma with tall cell features. Mod Pathol 25:144A

22. Dettmer MS, Schmitt A, Steinert H, Moch H, Komminoth P, Perren A (2012) Tall cell variant of papillary thyroid carcinoma- how many tall cells are needed? Mod Pathol 25:143

23. Morris LG, Shaha AR, Tuttle RM, Sikora AG, Ganly I (2010) Tall-cell variant of papillary thyroid carcinoma: a matched-pair analysis of survival. Thyroid 20:153-158

24. Lubitz CC, Economopoulos KP, Pawlak AC, Lynch K, Dias-Santagata D, Faquin WC, Sadow PM (2014) Hobnail variant of papillary thyroid carcinoma: an institutional case series and molecular profile. Thyroid 24:958-965

25. Nikiforov YE, Erickson LA, Nikiforova MN, Caudill CM, Lloyd RV (2001) Solid variant of papillary thyroid carcinoma: incidence, clinical-pathologic characteristics, molecular analysis, and biologic behavior. Am J Sur Pathol 25:1478-1484

26. Volante M, Collini P, Nikiforov YE, Sakamoto A, Kakudo K, Katoh R, Lloyd RV, LiVolsi VA, Papotti M, Sobrinho-Simoes M, Bussolati G, Rosai J (2007) Poorly differentiated thyroid carcinoma: the Turin proposal for the use of uniform diagnostic criteria and an algorithmic diagnostic approach. Am J Surg Pathol 31:1256-1264

27. Asioli S, Erickson LA, Righi A, Jin L, Volante M, Jenkins S, Papotti M, Bussolati G, Lloyd RV (2010) Poorly differentiated carcinoma of the thyroid: validation of the Turin proposal and analysis of IMP3 expression. Mod Pathol 23:1269-1278

28. Regalbuto C, Malandrino P, Tumminia A, Le Moli R, Vigneri R, Pezzino V (2011) A diffuse sclerosing variant of papillary th thyroid carcinoma: clinical and pathologic features and outcomes of 34 consecutive cases. Thyroid 21:383-389

29. Lam AK, Lo CY (2006) Diffuse sclerosing variant of papillary carcinoma of the thyroid: a 35-year comparative study at a single institution. Ann Surg Oncol 13:176-181

30. Sugitani I, Kasai N, Fujimoto Y, Yanagisawa A (2004) A novel classification system for patients with PTC: addition of the new variables of large ( $3 \mathrm{~cm}$ or greater) nodal metastases and reclassification during the follow-up period. Surgery 135:139-148

31. Lamartina L, Durante C et al (2015) Low risk differentiated thyroid cancer and radioiodine remnant ablation: a systematic review of the literature. J Clin Endocrinol Metab 100:1748-1761

32. Pacini $F$ et al (2016) Recommendations for post-surgical thyroid ablation in differentiated thyroid cancer: a 2015 position statement of the Italian Society of Endocrinology. J Endocrinol Investig 39:341-347
33. Castagna MG et al (2016) Reappraisal of the indication for radioiodine thyroid ablation in differentiated thyroid cancer patients. J Endocrinol Invest 39:1087-1094

34. Ruel E, Thomas S et al (2015) Adjuvant radioactive iodine therapy is associated with improved survival for patients with intermediate-risk papillary thyroid cancer. J Clin Endocrinol Metab 100:1529-1536

35. Pacini F, Ladenson PW et al (2006) Radioiodine ablation of thyroid remnants after preparation with recombinant human thyrotropin in differentiated thyroid carcinoma: results of an international, randomized, controlled study. J Clin Endocrinol Metab 91:926-932

36. Schlumberger M, Catargi B et al (2012) Strategies of radioiodine ablation in patients with low-risk thyoid cancer. NEJM 366:1663-1673

37. Mallick U, Harmer C et al (2012) Ablation with low-dose radioiodine and thyrotropin alfa in thyroid cancer. NEJM 366:1674-1685

38. Molinaro E, Giani C et al (2013) Patients with differentiated thyroid cancer who underwent radioiodine thyroid remnant ablation with low-activity ${ }^{131}$ I after either recombinant human TSH or thyroid hormone therapy withdrawal showed the same outcome after a 10-year follow-up. J Clin Endocrinol Metab 98:2693-2700

39. Verburg FA, Mader U et al (2014) Long term survival in DTC is worse after low-activity initial post-surgical I-131 therapy in both high and low risk patients. J Clin Endocrinol Metab 99:4487-4496

40. Castagna MG, Cevenini G et al (2013) Post-surgical thyroid ablation with low or high radioiodine activities results in similar outcomes in intermediate risk differentiated thyroid cancer patients. Eur J Endocrinol 169:23-29

41. Tala Jury HP, Castagna MG, Fioravanti C, Cipri C, Brianzoni E, Pacini F (2010) Lack of association between urinary iodine excretion and successful thyroid ablation in thyroid cancer patients. $J$ Clin Endocrinol Metab 95:230-237

42. American Thyroid Association Taskforce On Radioiodine Safety, Sisson JC, Freitas J, McDougall IR, Dauer LT, Hurley JR, Brierley JD, Edinboro CH, Rosenthal D, Thomas MJ, Wexler JA, Asamoah E, Avram AM, Milas M, Greenlee C (2011) Radiation safety in the treatment of patients with thyroid diseases by radioiodine 131I: practice recommendations of the American Thyroid Association. Thyroid 21(4):335-346

43. International Commission on Radiological Protection (2004) Release of patients after therapy with unsealed radionuclides. Ann ICRP 34(2):v-vi, 1-79

44. Raccomandazioni per la dimissione dei pazienti a seguito di terapia medico nucleare con 131I. http://www.fisicamedi ca.it/2014_07_01_Raccomandazioni_dimissione_pazienti_131I. pdf

45. Brassard M, Borget I, Edet-Sanson A, Giraudet AL, Mundler O, Toubeau M, Bonichon F, Borson-Chazot F, Leenhardt L, Schvartz C, Dejax C, Brenot-Rossi I, Toubert ME, Torlontano M, Benhamou E, Schlumberger M, THYRDIAG Working Group (2011) Long-term follow-up of patients with papillary and follicular thyroid cancer: a prospective study on 715 patients. J Clin Endocrinol Metab 2011 96:1352-1359

46. Miyauchi A, Kudo T, Miya A, Kobayashi K, Ito Y, Takamura Y, Higashiyama T, Fukushima M, Kihara M, Inoue H, Tomoda C, Yabuta T, Masuoka H (2011) Prognostic impact of serum thyroglobulin doubling-time under thyrotropin suppression in patients with papillary thyroid carcinoma who underwent total thyroidectomy. Thyroid 21:707-716

47. Durante C, Montesano T, Attard M, Torlontano M, Monzani F, Costante G, Meringolo D, Ferdeghini M, Tumino S, Lamartina L, Paciaroni A, Massa M, Giacomelli L, Ronga G, Filetti S, PTC Study Group (2012) Long-term surveillance of papillary thyroid cancer patients who do not undergo post-operative radioiodine 
remnant ablation: is there a role for serum thyroglobulin measurement. J Clin Endocrinol Metab 2012 97:2748-2753

48. Durante C, Montesano T, Torlontano M, Attard M, Monzani F, Tumino S, Costante G, Meringolo D, Bruno R, Trulli F, Massa M, Maniglia A, D’Apollo R, Giacomelli L, Ronga G, Filetti S, PTC Study Group (2013) Papillary thyroid cancer: time course of recurrences during postsurgery surveillance. J Clin Endocrinol Metab 2013 98:636-642

49. Castagna MG, Tala Jury HP, Cipri C, Belardini V, Fioravanti C, Pasqui L, Sestini F, Theodoropoulou A, Pacini F (2011) The use of ultrasensitive thyroglobulin assays reduces but does not abolish the need for TSH stimulation in patients with differentiated thyroid carcinoma. J Endocrinol Investig 34:e219-e223

50. Torlontano M, Crocetti U, Augello G, D'Aloiso L, Bonfitto N, Varraso A, Dicembrino F, Modoni S, Frusciante V, Di GA, Bruno R, Filetti S, Trischitta V (2006) Comparative evaluation of recombinant human thyrotropin-stimulated thyroglobulin levels, 131I whole body scintigraphy, and neck ultrasonography in the followup of patients with papillary thyroid microcarcinoma who have not undergone radioiodine therapy. J Clin Endocrinol Metab 2006 91:60-63

51. Tuttle RM, Tala H, Shah J, Leboeuf R, Ghossein R, Gonen M, Brokhin M, Omry G, Fagin JA, Shaha A (2010) Estimating risk of recurrence in differentiated thyroid cancer after total thyroidectomy and radioactive iodine remnant ablation: using response to therapy variables to modify the initial risk estimates predicted by the new American Thyroid Association staging system. Thyroid 20:1341-1349

52. Vaisman F, Tala H, Grewal R, Tuttle RM (2011) In differentiated thyroid cancer, an incomplete structural reponse to therapy is associated with significantly worse clinical outcomes than only an incomplete thyroglobulin response. Thyroid 21:1317-1322

53. Durante C, Haddy N, Baudin E, Leboulleux S, Hartl D, Travagli JP, Caillou B, Ricard M, Lumbroso JD, De Vathaire F, Schlumberger M (2006) Long-term outcome of 444 patients with distant metastases from papillary and follicular thyroid carcinoma: benefits and limits of radioiodine therapy. J Clin Endocrinol Metab 91:2892-2899

54. Robbins RJ, Wan Q, Grewal RK, Reibke R, Gonen M, Strauss HW, Tuttle RM, Drucker W, Larson SM (2006) Real-time prognosis for metastatic thyroid carcinoma based on 2-[18F]fluoro2-deoxy-D-glucose-positron emission tomography scanning. J Clin Endocrinol Metab 91:498-505

55. Vaisman F, Momesso D, Bulzico DA, Pessoa CH, da Cruz MD, Dias F, Corbo R, Vaisman M, Tuttle RM (2013) Thyroid lobectomy is associated with excellent clinical outcomes in properly selected differentiated thyroid cancer patients with primary tumors greater than $1 \mathrm{~cm}$. J Thyroid Res 2013:398194. https:// doi.org/10.1155/2013/398194

56. Momesso DP, Vaisman F, Yang SP, Bulzico DA, Corbo R, Vaisman M, Tuttle RM (2016) Dynamic risk stratification in patients with differentiated thyroid cancer treated without radioactive iodine. J Clin Endocrinol Metab 101(7):2692-2700

57. Freudenberg LS, Jentzen W, Petrich T, Frömke C, Marlowe RJ, Heusner T, Brandau W, Knapp WH, Bockisch A (2010) Lesion dose in differentiated thyroid carcinoma metastases after rhTSH or thyroid hormone withdrawal: 124I PET/CT dosimetric comparisons. Eur J Nucl Med Mol Imaging 37:2267-2276

58. Potzi C, Moameni A, Karanikas G, Preitfellner J, Becherer A, Pirich C, Dudczak R (2006) Comparison of iodine uptake in tumour and nontumour tissue under thyroid hormone deprivation and with recombinant human thyrotropin in thyroid cancer patients. Clin Endocrinol (Oxf) 2006 65:519-523

59. Rani D, Kaisar S, Awasare S, Kamaldeep P, Abhyankar A, Basu S (2014) Examining recombinant human TSH primed 131I therapy protocol in patients with metastatic differentiated thyroid carcinoma: comparison with the traditional thyroid hormone withdrawal protocol. Eur J Nucl Med Mol Imaging 41:1767-1780

60. Tala H, Robbins R, Fagin JA, Larson SM, Tuttle RM (2011) Fiveyear survival is similar in thyroid cancer patients with distant metastases prepared for radioactive iodine therapy with either thyroid hormone withdrawal or recombinant human TSH. J Clin Endocrinol Metab 96:2105-2111

61. Van Nostrand D, Atkins F, Yeganeh F, Acio E, Bursaw R, Wartofsky L (2002) Dosimetrically determined doses of radioiodine for the treatment of metastatic thyroid carcinoma. Thyroid 12:121-134

62. Deandreis D, Rubino C, Tala H, Leboulleux S, Terroir M, Baudin E, Larson S, Fagin JA, Schlumberger M, Tuttle RM (2017) Comparison of empiric versus whole-body/-blood clearance dosimetry-based approach to radioactive iodine treatment in patients with metastases from differentiated thyroid cancer. J Nucl Med 58:717-722

63. Pacini F, Lippi F, Formica N, Elisei R, Anelli S, Ceccarelli C, Pinchera A (1987) Therapeutic doses of iodine-131 reveal undiagnosed metastases in thyroid cancer patients with detectable serum thyroglobulin levels. J Nucl Med 28:1888-1891

64. Padovani RP, Robenshtok E, Brokinn M, Tuttle RM (2012) Even without additional therapy, serum thyroglobulin concentrations often decline for years after total thyroidectomy and radioactive remnant ablation in patients with differentiated thyroid cancer. Thyroid 22:778-783

65. Clement SC, Peeters RP, Ronckers CM et al (2015) Intermediate and long-term adverse effects of radioiodine therapy for differentiated thyroid carcinoma-a systematic review. Cancer Treat Rev 41:925-934

66. Dingle IF, Mishoe AE, Nguyen SA et al (2013) Salivary morbidity and quality of life following radioactive iodine for well-differentiated thyroid cancer. Otolaryngol Head Neck Surg 148:746-752

67. Canale D, Ceccarelli C, Caglieresi C et al (2015) Effects of radioiodine treatment for differentiated thyroid cancer on testis function. Clin Endocrinol (Oxf) 82:295-299

68. Wu JX, Young S, Ro K et al (2015) Reproductive outcomes and nononcologic complications after radioactive iodine ablation for well differentiated thyroid cancer. Thyroid 25:133-138

69. Khang AR, Cho SW, Choi HS et al (2015) The risk of second primary malignancy is increased in differentiated thyroid cancer patients with a cumulative (131) I dose over 37 GBq. Clin Endocrinol (Oxf) 23:220-227

70. Sgouros G, Kolbert KS, Sheikh A, Pentlow KS, Mun EF, Barth A, Robbins RJ, Larson SM (2004) Patient-specific dosimetry for 131I thyroid cancer therapy using 124I PET and 3-dimensionalinternal dosimetry (3D-ID) software. J Nucl Med 45:1366-1372

71. Brose MS, Nutting CM, Jarzab B et al (2014) Sorafenib in radioactive iodine-refractory, locally advanced or metastatic differentiated thyroid cancer: a randomised, double-blind, phase 3 trial. Lancet 384:319-328

72. Schlumberger M, Tahara M, Wirth LJ et al (2015) Lenvatinib versus placebo in radioiodine-refractory thyroid cancer. N Engl J Med 372:621-630

73. Abadin SS, Kaplan EL, Angelos P (2010) Malpractice litigation after thyroid surgery: the role of recurrent laryngeal nerve injuries, 1989-2009. Surgery 148(4):718-722

74. Polistena A, Di Lorenzo P, Sanguinetti A, Buccelli C, Conzo G, Conti A, Niola M, Avenia N (2016) Medicolegal implications of surgical errors and complications in neck surgery: a review based on the Italian current legislation. Open Med 2016 11:298-306

75. Randolph GW, Duh QY, Heller KS, LiVolsi VA, Mandel SJ, Steward DL, Tufano RP, Tuttle RM (2012) The prognostic significance of nodal metastases from papillary thyroid carcinoma can be stratified based on the size and number of metastatic lymph 
nodes, as well as the presence of extranodal extension. Thyroid 22:1144-1152

76. Leboulleux S, Rubino C, Baudin E, Caillou B, Hartl DM, Bidart JM, Travagli JP, Schlumberger M (2005) Prognostic factors for persistent or recurrent disease of papillary thyroid carcinoma with neck lymph node metastases and/or tumor extension beyond the thyroid capsule at initial diagnosis. J Clin Endocrinol Metab 90:5723-5729
77. Lango M, Flieder D, Arrangoiz R, Veloski C, Yu JQ, Li T, Burtness B, Mehra R, Galloway T, Ridge JA (2013) Extranodal extension of metastatic papillary thyroid carcinoma: correlation with biochemical endpoints, nodal persistence, and systemic disease progression. Thyroid 23:1099-1105

\section{Affiliations}

F. Pacini ${ }^{1}$ - F. Basolo ${ }^{2} \cdot$ R. Bellantone ${ }^{3} \cdot$ G. Boni ${ }^{4} \cdot$ M. A. Cannizzaro ${ }^{5} \cdot$ M. De Palma $^{6} \cdot$ C. Durante $^{7} \cdot$ R. Elisei $^{8}$.

G. Fadda ${ }^{9}$. A. Frasoldati ${ }^{10} \cdot$ L. Fugazzola ${ }^{11,12} \cdot$ R. Guglielmi ${ }^{13}$. C. P. Lombardi ${ }^{3} \cdot$ P. Miccoli ${ }^{2} \cdot$ E. Papini $^{13} \cdot$ G. Pellegriti $^{14}$. $^{2}$ L. Pezzullo ${ }^{15} \cdot$ A. Pontecorvi $^{16} \cdot$ M. Salvatori ${ }^{17} \cdot$ E. Seregni $^{18} \cdot$ P. Vitti $^{8}$

1 Department of Medical, Surgical and Neurological Sciences, University of Siena, 53100 Siena, Italy

2 Department of Surgical, Medical, Molecular Pathology and Critical Area, University of Pisa, Pisa, Italy

3 U.O.C. Chirurgia Endocrina e Metabolica, Fondazione Policlinico Universitario A. Gemelli, Università Cattolica del Sacro Cuore, Rome, Italy

4 Regional Center of Nuclear Medicine, University of Pisa, Pisa, Italy

5 Department of Medical and Surgical Sciences, Advanced Technologies "G.F.Ingrassia", University of Catania, Catania, Italy

6 Dipartimento Chirurgico Generale e Polispecialistico Chirurgia 2, AORN Cardarelli, Naples, Italy

7 Department of Internal Medicine and Medical Specialties, University of Rome Sapienza, Rome, Italy

8 Department of Clinical and Experimental Medicine, University of Pisa, Pisa, Italy

9 Institute of Pathology, Catholic University of the Sacred Heart, Rome, Italy

10 Endocrinology Unit, Arcispedale S. Maria Nuova-IRCCS, Reggio Emilia, Italy
11 Division of Endocrine and Metabolic Diseases, Istituto Auxologico Italiano IRCCS, Milan, Italy

12 Department of Pathophysiology and Transplantation, University of Milan, Milan, Italy

13 Department of Endocrinology, Regina Apostolorum Hospital, Albano Laziale, Italy

14 Endocrinology, Department of Clinical and Experimental Medicine, University of Catania, Garibaldi-Nesima Medical Center, Catania, Italy

15 Thyroid and Parathyroid Surgery Unit, Istituto Nazionale per lo Studio e la Cura dei Tumori-IRCCS 'Fondazione G. Pascale', Naples, Italy

16 Cattedra di Endocrinologia, Area di Endocrinologia e Malattie Metaboliche, Università Cattolica del Sacro Cuore, Rome, Italy

17 Istituto di Medicina Nucleare, Policlinico Gemelli, Rome, Italy

18 Struttura di Terapia Medico Nucleare ed Endocrinologia U.O. Medicina Nucleare Fondazione IRCCS Istituto Nazionale dei Tumori Milano, Milan, Italy 